\title{
Analysis of factors affecting wireless communication systems in underground coal mines
}

David P. McGraw

West Virginia University

Follow this and additional works at: https://researchrepository.wvu.edu/etd

\section{Recommended Citation}

McGraw, David P., "Analysis of factors affecting wireless communication systems in underground coal mines" (2008). Graduate Theses, Dissertations, and Problem Reports. 1979.

https://researchrepository.wvu.edu/etd/1979

This Thesis is protected by copyright and/or related rights. It has been brought to you by the The Research Repository @ WVU with permission from the rights-holder(s). You are free to use this Thesis in any way that is permitted by the copyright and related rights legislation that applies to your use. For other uses you must obtain permission from the rights-holder(s) directly, unless additional rights are indicated by a Creative Commons license in the record and/ or on the work itself. This Thesis has been accepted for inclusion in WVU Graduate Theses, Dissertations, and Problem Reports collection by an authorized administrator of The Research Repository @ WVU. For more information, please contact researchrepository@mail.wvu.edu. 
Analysis of Factors Affecting Wireless Communication Systems in Underground Coal Mines

David P. McGraw

\author{
Thesis submitted to the \\ College of Engineering and Mineral Resources \\ at West Virginia University \\ in partial fulfillment of the requirements \\ for the degree of
}

\author{
Master of Science \\ in \\ Mining Engineering
}

Dr. A. Wahab Khair

Dr. Syd S. Peng Jim Dean M.S.

\author{
Department of Mining Engineering \\ Morgantown, West Virginia \\ 2008
}

Keyword: Underground Coal Mine Wireless Communications, Mining

Engineering, Coal Mine Safety 


\section{ABSTRACT}

\section{Analysis of Factors Affecting Wireless Communications Systems in Underground Coal Mines}

\section{David P. McGraw}

Since recent disasters in the mining industry in 2006 the push for a reliable communication system has became a high priority. This thesis will discuss each "wireless" communication system available and/or being developed for underground mines as well as the frequencies that will be used for each system's operation.

The second part of the investigation will be on the factors affecting these frequencies as well as possible ways to model these factors analytically. It is important to understand these factors in order to make intelligent conclusions as to what communication systems will perform adequately in an underground mining environment.

The third area discussed will be an analysis of empirical studies completed by various agencies, companies, and schools (including West Virginia University). These studies were completed to judge the practicality and performance of each system. Practicality is important to the mining industry. The industry tends not to accept new technology quickly, so proof of performance is a key component to introducing the products into the market. 


\section{ACKNOWLEDGEMENTS}

The author of this thesis would like to express his sincere appreciation to those who have contributed to the contents of this thesis. Particular thanks go to:

Professor Dr. A. Wahab Khair, thesis advisor, for his guidance, encouragement, assistance, and advice during the development of this thesis;

The West Virginia University, Department of Mining Engineering and the West Virginia Coal and Energy Research Bureau (WVCERB) for financial support of this research; 


\section{Table of Contents}

Chapter 1 Problem Statement............................................................... 1

Chapter 2 Objective ....................................................................... 2

Chapter 3 Literature and Technology Review........................................... 3

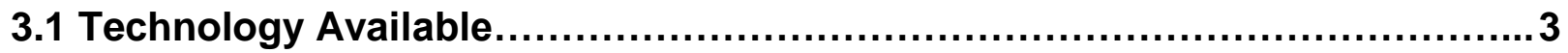

3.1.1 In-Mine Wireless Systems...................................................... 4

3.1.1.1 UHF / VHF Systems............................................................... 5

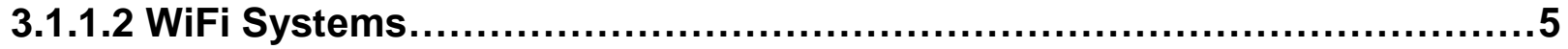

3.1.1.3 Medium Frequency Systems....................................................

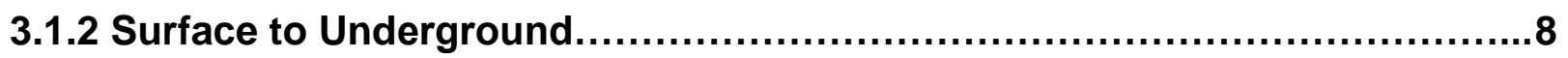

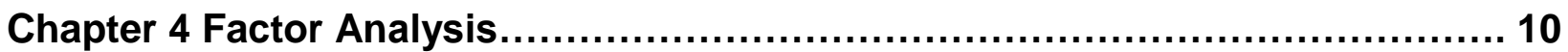

4.1 Coal Properties and Mine Entry Environment................................... 10

4.2 VHF / UHF / WiFi Propagation Model.............................................. 11

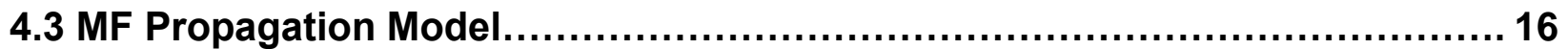

4.3.1 Transmission Line Modeling .......................................................

4.3.1.1 Single Conductor Transmission Line.......................................... 17

4.3.1.2 Multi-Conductor Transmission Line........................................... 20

4.4 Raytheon's Survivability Analysis of Buried Communication Cable..............20

4.5 ELF / VLF Propagation Modeling .................................................... 23

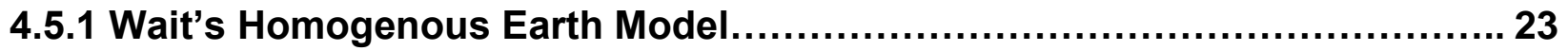

4.5.2 Shope's Three-Layered Earth Model............................................... 29

Chapter 5 Empirical and Practical Tests............................................ 32

5.1 WVU Test at Lake Lynn Laboratory.............................................. 32

5.2 Foundation Coal Leaky Feeder Analysis........................................... 33

5.3 Leaky Feeder Explosion Test....................................................... 34

5.4 MSHA Wireless Communication Study............................................ 37

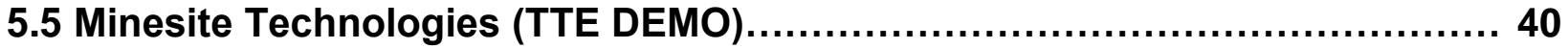

Chapter 6 Conclusions and Recommendations....................................... 41

6.1 Recommendation of a Risk Assessment for Battery Back-ups..................41

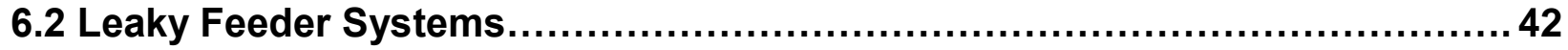

6.3 Medium Frequency Systems............................................................ 43 
6.4 Through the Earth Systems....................................................... 44

Appendix A-1 MSHA Approved Wireless Communication Devices (2008).......... 46

Appendix A-2 Miner Act (2006) ........................................................ 48

Appendix A-3 Wireless Communication requirments in the state of WV............ 49

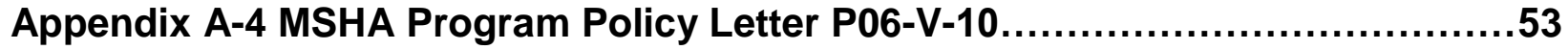

Appendix A-5 MSHA Program Policy Letter P08-V-10 ..............................54

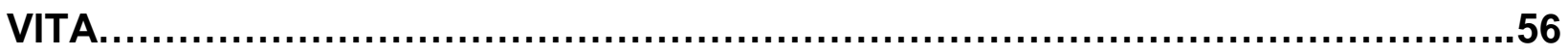

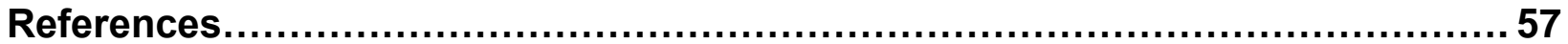




\section{List of Figures}

Figure 3-1. Systems operating frequencies............................................. 4

Figure 4-1. Plane waves incident on tunnel wall...................................... 12

Figure 4-2. The coal mine tunnel dimensions........................................ 12

Figure 4-3. The sum of path losses...................................................... 15

Figure 4-4. Model Geometry for cable burial analysis.............................22

Figure 4-5. Preliminary Results from Varying Trench Width........................22

Figure 4-6. Preliminary Results from Varying Burial Depth......................... 23

Figure 4-7. A current loop in an isotropic homogeneous earth....................... 24

Figure 4-8. Magnitude of the magnetic field directly below a loop................. 27

Figure 4-9. Vertical component of magnetic field as function of distance from the

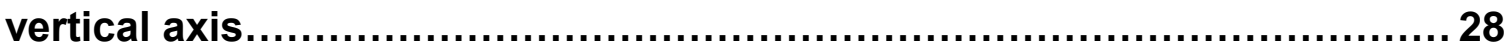

Figure 4-10. Horizontal component of magnetic field as function of distance from

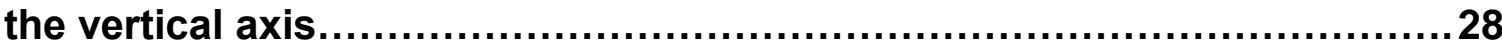

Figure 4-11. Side elevation view of the three-layer earth model...................... 29

Figure 5-1. Pre-Blast Picture of a leaky feeder cable hung parallel to entry..........35

Figure 5-2. Post Blast Picture of a leaky feeder cable hung parallel to entry........35

Figure 5-3. Pre-Blast Picture of a leaky feeder cable hung perpendicular to

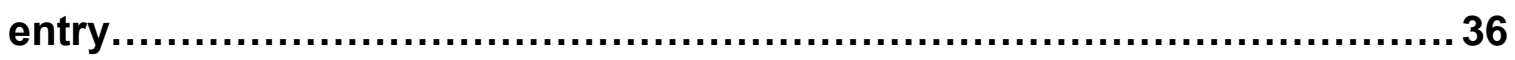

Figure 5-4. Post Blast Picture of a leaky feeder cable hung perpendicular to

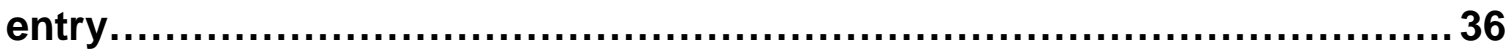

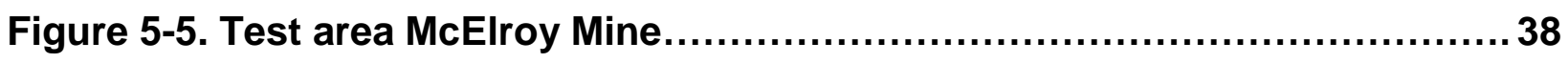




\section{Chapter 1}

\section{Problem Statement}

Currently mine communication systems have been relatively low tech in comparison to the systems currently in place on the surface. Generally the communication usually consists of a twisted pair wired page phone system or possible if rail is used in the mine trolley phones are used. However, past experience has shown that in the event of a fire, explosion, or major roof fall these type of systems do not perform adequately.

The need for survivable mine tracking and emergency communication systems has been prompted by recent mine disasters in the United States, namely

- Coal mine water inundation, Quecreek mine, PA, 2002

- Mine explosion in Sago mine, WV. 2006

- Mine Collapse in Crandle Canyon, UT, 2007

More than a hundred systems or components have been or are being developed to serve in case of events similar to the ones listed above. However the design of these systems has become a task of great difficulty due to the mining environment not being conducive to typical radio frequencies that work well on the surface. The factors affecting these frequencies differ from band to band. The mining environment also provides challenges to devices that could cause system failure in the event of an accident. It is important to understand these factors and challenges in order to make intelligent conclusions as to what communication systems will perform adequately in an underground mining environment during emergency and non-emergency situations. These need to be evaluated before they are installed throughout the USA. 


\section{Chapter 2}

\section{Objective}

The objective of this research is to evaluate wireless emergency communication systems that will be used in underground coal mines. A thorough analysis of several principles of each communication system and wave transmission mechanisms associated with each system has been studied and each system has been evaluated based on the following:

- Ground penetrating efficiency,

- Transmission distance,

- Survivability,

- Upgrading of system,

- Maintenance.

These are the principles that have been chosen to evaluate the performance of the new communication systems and will guide the direction of this thesis.

In the conclusion of this thesis, recommendations of future research as well as statements of fact will be made that should be considered when deciding what communication is appropriate for mine operation communication as well as emergency communications. 


\section{Chapter 3}

\section{Literature and Technology Review}

For the initial steps of this research, a look at the technology being developed as well as the currently approved wireless systems and the literature that surrounds each of them has been done. By analyzing these systems one can find out what has been done as well as what needs to be done to improve miner communications and mine safety. Following the literature and technology survey, this research will produce conclusions and recommendations regarding the technological status of wireless communications in underground coal mines.

\subsection{Technology Available}

The wireless communication systems currently available can be split into two different types of systems.

- Through the earth (TTE).

- In mine radio systems.

These systems actually operate at different frequencies, but may be integrated together.

Figure 3-1 shows what frequencies each system operates at as well as their corresponding wavelengths. Described in the following sections are the systems that are currently being developed as well as the systems that are currently approved for use in underground coal mines. 


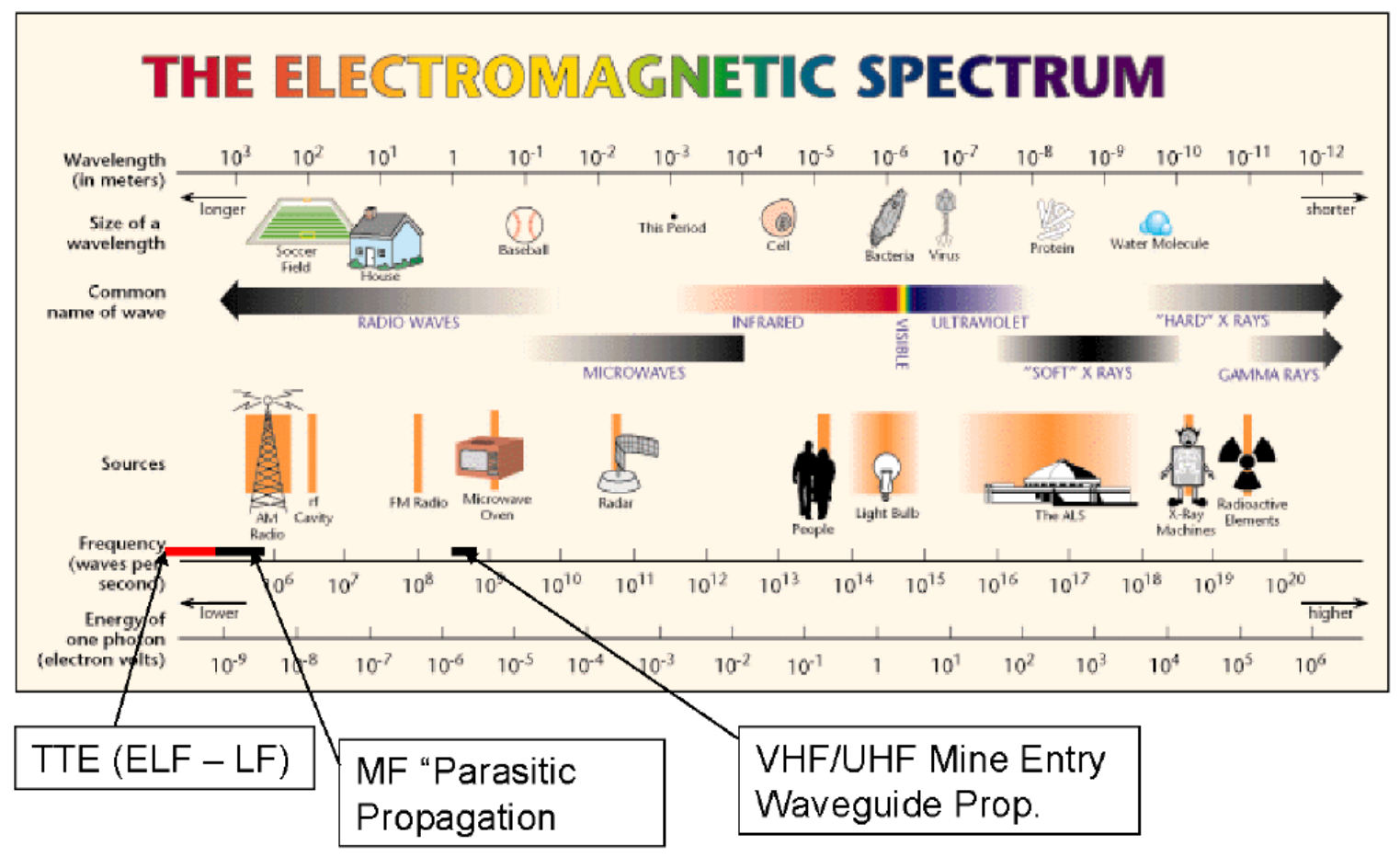

Figure 3-1 Mine communication systems operating frequencies.

\subsubsection{In-Mine Wireless Systems}

Frequency selection has a great impact on the overall hardware architecture of the system as well as the mechanics of the wireless signal propagation from the transmitter to the receiver. Some frequencies utilize the coal mine entry as a waveguide, enhancing signal propagation, while other frequencies will not travel more than $50 \mathrm{ft}$ in a bare coal mine entry. Unaided transmitted radio signals in a certain frequency ranges (WiFi or UHF) may travel (line-of-sight) up to $1000 \mathrm{ft}$. and still be received, but typically the signal will not be received if the signal is forced to turn corners for more than two crosscuts. Other types of communication signals (medium frequencies) utilize parasitic propagation. Parasitic propagation works in the proximity of wires, conductors, pipes, and rails that can enhance the propagation of signals at 
certain frequencies. Both types of systems will be discussed further later in this thesis. The most common systems available are hand held two-way radios in conjunction with a corresponding leaky feeder backbone or various "breadcrumb" type repeaters, scattered strategically throughout the mine. However in the event of a disaster such as an explosion, roof fall, or mine fire these types of infrastructures are susceptible to damage that could result in the loss of communication. It is the goal of this research to find the optimum system to use in an underground coal mine.

\subsubsection{UHF / VHF Systems}

Ultra High Frequency (UHF) / Very High Frequency (VHF) systems are currently the most available systems for use in underground coal mines. These systems typically operate at approximately $450 \mathrm{MHz}$ for UHF and $150 \mathrm{MHz}$ for VHF. They use hand-held radios and are usually accompanied by a leaky feeder system. A leaky feeder system consists of a surface base station and "lossy" coaxial cable strung along the roof throughout the mine. The cable is usually accompanied by in line amplifiers and or repeaters to boost the signal strength and enhance the signal quality. Leaky feeder systems can be expensive due to the high cost of the specialized cable and hardware that is needed to make the system operate properly.

\subsubsection{WiFi Systems}

WiFi is denoted for 'wireless fidelity', a term for wireless local area networks conforming to a protocol specified in IEEE 802.11. This technology used to meet the needs of underground coal mining is referred to as "Wireless Mesh Networking." The 
new IEEE 802.11s standard will enable a collection of Wireless Access Points (WAP) to be interconnected with wireless links that enable automatic topology learning and dynamic path configuration.

Wireless modems (sometimes called "Hot Spots" or nodes) are strategically placed throughout a work area, and each unit can receive, transmit, or act as a signal repeater. This multi-hop style network can be designed to be redundant and automatically configures itself and also has a "learning" and "self-healing" capability. There are no predefined signal pathways between the nodes. Failure of any one node or closure of any one signal path (due to loss of power or an event such as a fire or a roof fall) has little impact on the whole network (Schiffbauer, 2006). The application of this type of network could greatly enhance the reliability of a wireless coal mine communication network. Still, if all possible radio signal paths are closed or if too many nodes fail, communications will stop.

These types of systems are currently being developed right now using various frequencies. One of the main frequencies being considered is $2.4 \mathrm{GHz}$. It is currently the most popular mostly because of the availability of hardware that has already been developed for surface and other applications. Another frequency being developed is $900 \mathrm{MHz}$. This frequency is being considered because of its wave propagation performance in underground coal mine entries. Wave propagation of various frequencies will be discussed in the analytical study portion of this thesis. 


\subsubsection{MF Systems}

While not yet fully developed, research has shown that medium frequencies (MF) offer a viable approach to underground communications in both coal and metal/nonmetal mines under certain circumstances. MF transmission is feasible for both personnel and vehicular communications. It does not experience the same attenuation characteristics and severe corner losses of UHF and WiFi communications; nor does it require the use of expensive leaky feeder cable. Furthermore, it does not experience the high noise levels of Low Frequency communication systems. Research has demonstrated ranges of $1000-1500 \mathrm{ft}$. in conductor-free areas, and 1-2 miles in the presence of conductors (Schiffbauer, 2006).

Underground tests have shown that MF signals have the capability to inductively couple into, and reradiate from, continuous electrical conductors in such a way that these conductors become transmission lines or an antenna system for the signals (Stolarczyk, 1980). The existence of electrical conductors in the entryway provides the means for what is referred to as the "parasitic propagation mode" of radio signal propagation in an underground mine. Testing has shown that MF signals could be carried out by magnetically inducing a signal current flow on other nearby conductor (Stolarcyk, 1980). Thus all of the entryway conductors could provide a means of minewide signal distribution. The method emulates the general properties of a leaky feeder without requirement of the specialized cable. USBM testing has also shown that MF radio signal propagation was possible in "natural waveguides" existing in certain layered formations (Sacks and Chufo, 1978). There have been a few commercially developed walkie-talkie systems that take advantage of this property. One vendor (Conspec) has a 
permissible system available (Schiffbauer, 2006). Also a vendor known as (Kutta) is currently developing a MF system that utilizes repeaters as well as hand held radios (Chirdon, 2006). The range depends on a number of factors which include conductivity of the surrounding strata, type of floor and roof, distance to conductors, type of conductors, etc. The size of the antenna can also be cumbersome.

\subsubsection{Surface to Underground}

Radio communications in the frequency band $1 \mathrm{kHz}-10 \mathrm{kHz}$ offers the potential for wireless communications through-the-earth (TTE). Most commercial TTE systems provide communication from the surface to underground only. This is because relatively high power is needed to transmit through considerable distances from the surface to the workers in the mine. The use of high power systems underground may not be safe, especially in emergency situations in which methane gas or coal dust has accumulated. Obviously those types of situations could make for a potentially explosive environment. MineSite Technologies has developed a prototype two-way text communication system that has proven itself to work in ranges of overburden up to $1800 \mathrm{ft}$. One proposed method of two-way communications, by MineSite Technologies, is to use this system as a fixed-station / intermediate relay unit located in a safe area, such as a mine refuge chamber, underground away from the mining operations. The miner could then use a mobile UHF radio to communicate directly with the relatively close underground relay. The relay receives the signal then rebroadcast an ELF signal to communicate with the surface unit. However the performance of the ELF portion of the system is not 
always reliable and consistent since the system is very susceptible to electromagnetic noise. 


\section{Chapter 4}

\section{Factor Analysis}

It is the goal of this research to analyze and model the mine environment with respect to electromagnetic signals as well as other factors that affect the communication systems described in Chapter 3. Furthermore it is also within the aspiration of this thesis to use the data acquired to discover the optimal system for communication in an underground coal mine. The use of mathematics and fundamental physics will be used to describe and model the electromagnetic wave propagation as well as the factors that affect it. This analysis will be considered and compared in conjunction with the empirical data that has been collected and discussed in Chapter 5. What's more the two analyses will be used to make recommendations concerning what communication system will work the best for each individual mine in Chapter 6 .

\subsection{Coal Properties and Mine Entry Environment}

In order to do a complete analysis one must first investigate the environment. A typical coal mine entry is rectangular with a height of 5-7 feet and a width of 16-21 feet. Tracks are sometimes seen at the bottom of the portal with a track separation of 3 feet for man trip vehicles or supply trains. Trolley line power cable usually accompanies the track. It can be seen at the top of the entry along with other various conductors such as aluminum water pipe and a page phone line. Also seen on the roof of the mine entry are roof bolts, straps and a wire mesh for roof control.

The electrical properties of coal are discussed in the literature. Chufo et al (Chufo, 1977) gives values of $5 \times 10^{-4}$ to $2 \times 10^{-2} \mathrm{~S} / \mathrm{m}$ (Siemens per Meter) for the 
electrical conductivity of coal. J. Cook (Cook, 1970) gives electrical resistivites of $500-2000$ ohms-meters at $100 \mathrm{MHz}$ with a scaling of $1 / f$ at lower frequencies. This corresponds to the electrical conductivities of $5 \times 10^{-6}$ to $2 \times 10^{-5} \mathrm{~S} / \mathrm{m}$ at a frequency of $f \sim 1 \mathrm{MHz}$. Cook also gives a relative dielectric constant for coal of $\varepsilon_{r} \sim 2-4$.

\subsection{VHF / UHF / WiFi Signal Propagation Model}

For most In-mine wireless communication systems the use of the certain frequencies, being transmitted from a hand held radio, propagating down a mine entry is the beginning of the communication systems operation. The attenuation of these signals due to absorption, reflection, and refraction is the main reason that the hand held radios only reach so far. Figure 4-1 shows an EM wave's incident on a wall or rib of the mine entry. A portion of the energy is assumed to be reflected and a portion is refracted into the entry wall. The wave is attenuated as it propagates down the entry, repeatedly being reflected off walls, floor, and ceiling. The wave loses energy at each reflection as a portion of the energy is carried away in the transmitted wave.

To model this, this research was based on the work of Emslie et al (1975). They solve Maxwell's equations for an EM wave propagating in the z-direction for a mine entry of dimensions shown in Figure 4-2. 


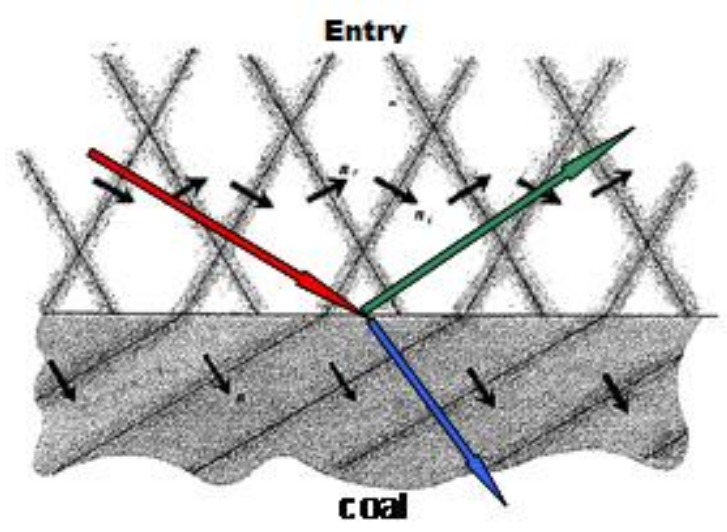

Figure 4-1. Plane waves incident on entry wall (red) are specularly reflected (green) and a portion of the energy is transmitted (blue) into the entry wall.

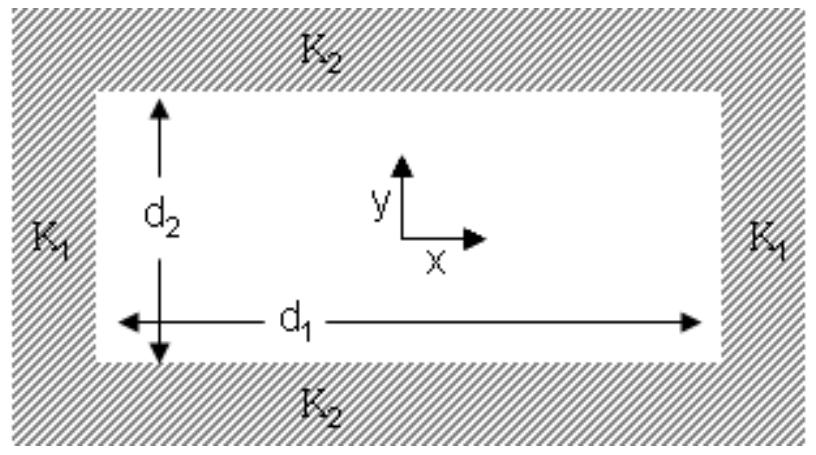

Figure 4-2. The coal mine entry has dimensions $d_{1} X d_{2}$, with the walls having dielectric constants of $K_{1}$ and the floor and ceiling of $K_{2}$. 
The result for path loss, $L_{E h}$ in $d B$ for horizontally polarized waves is given by

$$
\begin{aligned}
& L_{E h}=4.34 \lambda^{2} z\left(\frac{K_{1}}{d_{1}^{3}\left(K_{1}-1_{-}^{-/ 2}\right.}+\frac{1}{d_{2}^{3}\left(K_{2}-1_{-}^{-/ 2}\right.}\right) \\
& \text { and } \\
& L_{E v}=4.34 \lambda^{2} z\left(\frac{1}{d_{1}^{3}\left(K_{1}-1_{-}^{-1 / 2}\right.}+\frac{K_{2}}{d_{2}^{3}\left(K_{2}-1_{-}^{-1 / 2}\right.}\right)
\end{aligned}
$$

where $L_{E v}$ is for vertically polarized waves, $\lambda$ is the wavelength $m, K_{1}$ and $K_{2}$ are the relative dielectric constants for the walls $\left(K_{1}\right)$ and the floor and ceiling $\left(K_{2}\right)$ and $d_{1}$ and $d_{2}$ (meters) are the width and height of the entry respectively. The path loss is linear with distance and depends on the square of the wavelength and the inverse cube of the entry dimensions, $\lambda^{2} / d^{3}$.

Further on in the work of Emslie et al., (1975) they proceeded to approximate the effects of rib roughness which in addition to reflection, permits a fraction of the energy to be diffusely dispersed with energy being carried by de-polarized waves. Roughness (h), is the standard deviation from the mean of the surface and is in meters.

Emslie et al, (1975) also accounted for the possibility of wall and ceiling tilt (in the sense of making the rectangular cross-section larger or smaller as it progresses down the $z$-axis) through an angle $(\theta)$ in radians, and the antenna insertion loss $C$ (in $\mathrm{dB}$ ), from inefficient coupling of the radio antenna to the waveguide mode. Lastly, Emslie et al, (1975) derived an expression for the path loss in propagation around a corner such as a cross-cut.

The equations for the path loss due to roughness, tilt, and antenna insertion (for an antenna located at the center of the entry) are respectively: 


$$
\begin{aligned}
& L_{\text {roughness }}=4.34 \pi^{2} h^{2} \lambda z\left(\frac{1}{d_{1}^{4}}+\frac{1}{d_{2}^{4}}\right) \\
& L_{\text {tilt }}=4.34 \pi^{2} \theta^{2} z\left(\frac{1}{\lambda}\right) \\
& \text { and } \\
& C=\frac{377 \lambda^{2}}{73 \pi^{2} d_{1} d_{2}}
\end{aligned}
$$

Figure 4-3 shows the results for the path loss as a function of frequency when combining the effects of refraction, and wall roughness and tilt, for an entry $5 \mathrm{~m}$ wide, 2 $\mathrm{m}$ high, wall roughness of $10 \mathrm{~cm}$ and relative dielectric constant for walls and ceiling and floor of 5. A sharp rise in the path loss occurs as the frequency decreases towards the cutoff frequency. For the larger values of tilt angle, there is a broad minimum in the path loss suggesting an optimum frequency. The minimum in the curve will shift depending on the cross-sectional dimensions of the coal mine.

The work of Emslie et al, (1975) has been compared to measured data in Goddard, (1973). Measurements were taken at $200 \mathrm{MHz}, 415 \mathrm{MHz}$, and $1000 \mathrm{MHz}$ in a entry $14 \mathrm{ft}$ wide and $7 \mathrm{ft}$ high for vertical and horizontal and cross-polarizations. The predictions for path loss per $100 \mathrm{ft}$ are in good agreement with the data for entry distances ranging from $100 \mathrm{ft}$ to several thousand feet. 


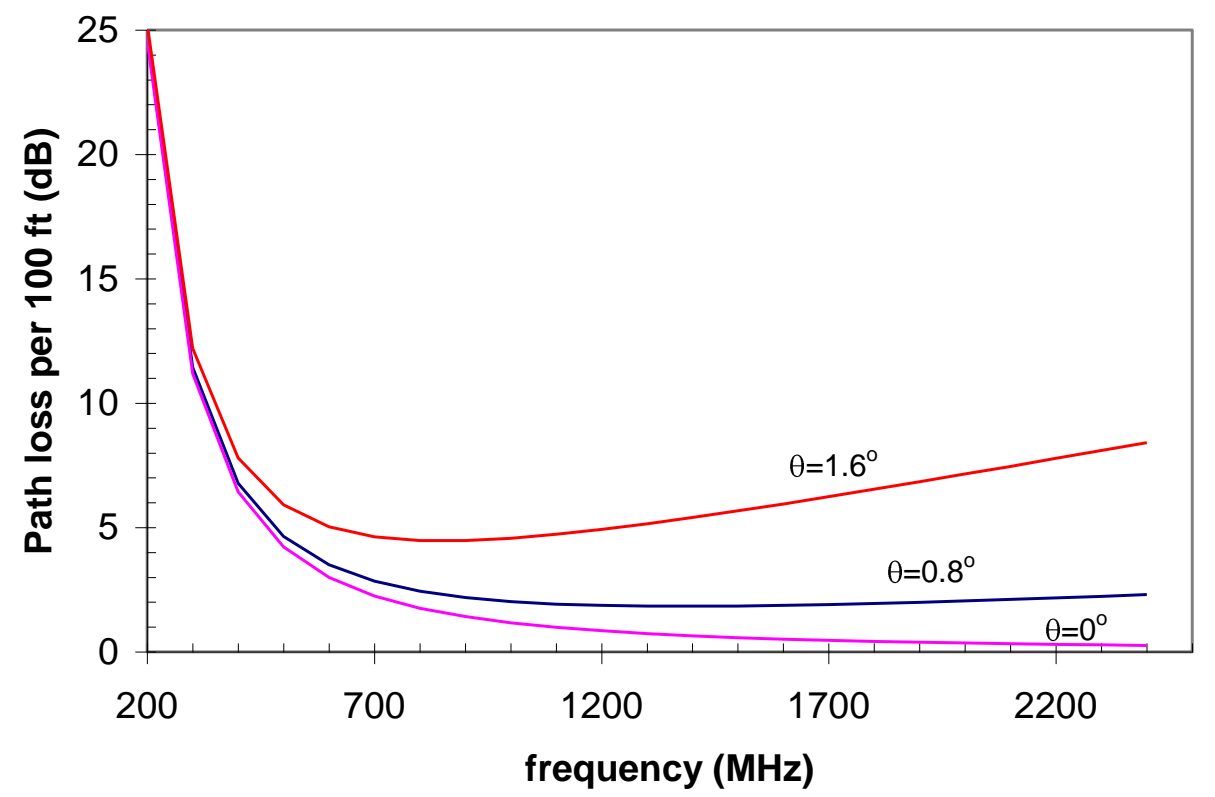

Figure 4-3. The sum of path losses for refraction, wall roughness, and tilt angle, as a function of frequency for various values of tilt $(\theta)$.

There are a number of issues that are not addressed by the model above and should be considered in further analyses.

- Although cross-cuts have little impact on the propagation losses down a given entry, it is important to communicate down those cross cuts. Introducing reflectors or repeaters at intersections will impact the losses in the main tunnel.

- This research also did not considered entries which have curves in them. The curve will disturb the normal waveguide behavior and likely increase the attenuation.

- Blocked or partially obscured entries (by equipment, belt structure, or etc.) have also not been considered. 


\subsection{Medium Frequency Propagation Model}

In the MF frequency range, two mechanisms have been suggested for the propagation of electromagnetic signals in coal mines. The first is a waveguide mode where an EM signal is propagated in a coal seam trapped between two layers of rock much like the model of UHF / VHF explained above. The second mode is a transmission line or parasitic coupling mode where electromagnetic signals couple to accidental or deliberate transmission line structures and then travel down the entry with relatively little attenuation (Chufo et al, 1977),(Stolarczyk, 1980).

Given that the transmission line mode of propagation appears to be the most useful for both parasitic and deliberate coupling of MF frequencies in coal mines, it was determined that the use of a transmission line model should be used to predict the propagation of a MF signal into a coal mine entry. A transmission line model predicts the voltages and currents on conductors as a function of position along the transmission line with respect to a common return or reference conductor. For example, a coaxial cable and a two wire TV antenna cable are both considered to be a single conductor (plus return) transmission line. A shielded twisted pair is considered to be a two conductor transmission line with the shield forming the return.

For this model, the coupling of electromagnetic energy to the transmission line and then coupling the propagated energy to a receiving antenna was not taken into consideration. That could be a topic of future research and will be recommended in Chapter 6. 


\subsubsection{Transmission Line Modeling}

The theory of transmission line modeling is discussed in Taylor (1965), McCray (1973), Smith (1983), Vance (1978), Paul (1992), and Paul (1994). Transmission line modeling allows us to use differential equations to predict voltages and currents at unit lengths of cable. This allows us to predict the magnitude of energy transmitted from one point on the line to another. The single conductor (plus return) transmission line first followed by the multi-conductor transmission line will be considered.

\subsubsection{Single Conductor Transmission Line}

The voltage $V$ and current $I$ on a single conductor transmission line is characterized by inductance per unit length $L$, capacitance per unit length $C$, resistance per unit length $R$, and conductance per unit length $G$. This relationship is described by the following equations:

$$
\begin{aligned}
& \frac{\partial V}{\partial x}+L \frac{\partial I}{\partial t}+R I=V_{g} \\
& \frac{\partial I}{\partial x}+C \frac{\partial V}{\partial t}+G V=I_{g}
\end{aligned}
$$

Where $V_{g}(x), I_{g}(x)$ are distributed voltage and current sources with units of volts per meter or amperes per meter, respectively. From these equations it can be derived that the complex propagation constant is;

$$
\gamma=\sqrt{Z Y}
$$

and the complex characteristic or surge impedance

$$
Z_{o}=\sqrt{Z / Y}
$$


The transmission line complex propagation constant can be expanded into real and imaginary parts, i.e.

$$
\alpha+i \beta=\sqrt{R+i \omega L \quad G+i \omega C}
$$

For an ideal transmission line $R=G=0$ dielectric we have

$$
\begin{aligned}
& \alpha=0 \\
& \beta=\omega \sqrt{L C}=\frac{\omega}{v}
\end{aligned}
$$

where $v=1 / \sqrt{L C}$. Likewise the characteristic impedance reduces to

$$
Z_{o}=\sqrt{L / C}
$$

For this special case the complex propagation constant is given by $\gamma=i \beta=i \omega / v$ and the useful set of conversion relationships can be developed

$$
\begin{aligned}
& Z_{o}=\sqrt{L / C}=L v=1 / C v \\
& v=1 / \sqrt{L C}=Z_{o} / L=1 / Z_{o} C \\
& L=Z_{o}^{2} C=Z_{o} / v=1 / v^{2} C \\
& C=L / Z_{o}^{2}=1 / Z_{o} v=1 / v^{2} L
\end{aligned}
$$

For example for a $Z_{o}=50 \Omega$ transmission line having a propagation velocity equal to $2 \times 10^{8} \mathrm{~m} / \mathrm{s}$ we have $L=0.25 \mu \mathrm{H} / \mathrm{m}$ and $C=100 \mathrm{pF} / \mathrm{m}$.

The solution of the homogeneous equations (3) is given by

$$
\begin{aligned}
& V(x)=A e^{\gamma x}+B e^{-\gamma x}=\bar{V}(x)+\vec{V}(x) \\
& I(x)=\frac{-A}{Z_{o}} e^{\gamma x}+\frac{B}{Z_{o}} e^{-\gamma x}=\frac{-\bar{V}(x)}{Z_{o}}+\frac{\vec{V}(x)}{Z_{o}}
\end{aligned}
$$

where the negative exponents correspond to waves traveling from left to right (in the positive $\mathrm{x}$ direction) and the positive exponents correspond to waves traveling from right to left. 
For a finite transmission line of length $l$, characteristic impedance $Z_{o}$, load impedance (receiver) $Z_{L}$ and source impedance (transmitter) $Z_{S}$ the terms of particular interest are the input impedance of the line and the input/output voltages for a specified Thevenin source voltage $V_{s}$. Where, $V_{s}$ is the voltage delivered to the transmission line by the radio signal.

Input impedance

$$
Z_{\text {in }}=Z_{o} \frac{1+\eta_{2} e^{-2 \gamma l}}{1-\eta_{2} e^{-2 \gamma l}}
$$

Input voltage $(x=0)$, would be the voltage at the point where the signal is coupled to the line

$$
V(0)=V_{S}\left(\frac{Z_{o}}{Z_{S}+Z_{o}}\right)\left(\frac{1+\eta_{L} e^{-2 \gamma l}}{1-\eta_{S} \eta_{L} e^{-2 \gamma l}}\right)
$$

Output voltage $(x=l)$, would be the voltage at the point where the signal is coupled to the receiver from the line

$$
V(l)=V_{S}\left(\frac{Z_{o}}{Z_{s}+Z_{o}}\right)\left(\frac{1+\eta_{L} e^{-\gamma l}}{1-\eta_{S} \eta_{L} e^{-2 \gamma l}}\right)
$$

where

$$
\begin{gathered}
\eta_{S}=\frac{Z_{s}-Z_{o}}{Z_{s}+Z_{o}} \\
\eta_{L}=\frac{Z_{L}-Z_{o}}{Z_{L}+Z_{o}}
\end{gathered}
$$




\subsubsection{Multi-conductor Transmission Line}

The extension of the single conductor plus return transmission line problem to the multi-line transmission line problem is formally straight forward, by replacing the transmission line voltage and currents $V$ and $I$ with voltage and current vectors $\bar{V}$ and $\bar{I}$ where $V_{i}(x), I_{i}(x)$ are the voltages and currents on the $i^{\text {th }}$ conductor at location $x$. It is important to note that the voltages are measured with respect to the reference conductor. To get the differential voltage between the $i^{\text {th }}$ and $j^{\text {th }}$ conductors the difference, i.e. $\Delta V_{i j}(x)=V_{i}(x)-V_{j}(x)$ is needed. With this in mind the differential equations for the multi-line problem are given by

$$
\begin{aligned}
& \frac{\partial \bar{V}}{\partial x}+L \frac{\partial \bar{I}}{\partial t}+R \bar{I}=\bar{V}_{g} \\
& \frac{\partial \bar{I}}{\partial x}+C \frac{\partial \bar{V}}{\partial t}+G \bar{V}=\bar{I}_{g}
\end{aligned}
$$

where $L, R, C, G$ are now the inductance, resistance, capacitance, and conductance matrices (per unit length), respectively and $\bar{V}_{g}(x), \bar{I}_{g}(x)$ are distributed voltage and current vectors, respectively

\subsection{Raytheon's Survivability Analysis of Buried Communication Cable}

Since MF communications could potentially utilize a buried twisted pair transmission line there has been some interest on the survivability of that line in the event of a disaster. One suggestion has been to bury the cable in a shallow trench inside the mine entry. It is the hope that by doing so the cable would be protected from roof falls. Raytheon has conducted a finite element analysis of factors affecting the 
theoretical survivability of a buried communications cable in a mine floor when a roof fall occurs. The geotechnical modeling software used for this exercise is FLAC, a finite difference modeling approach. To protect against $90 \%$ of all roof falls, $16,000 \mathrm{ft}-\mathrm{lb} / \mathrm{ft}$ of kinetic energy from a 6 foot fall is used as the basis for all of the models, creating a worst case scenario. A small velocity was initially applied to the surface of the FLAC model, and forces were recorded until the calculated force applied was attained. To determine the force that a roof fall would create, impulse momentum relationships were used (Terreri, 2008).

$$
\begin{aligned}
& M V+F(t)=M \\
& \text { Where, } \quad M=\text { mass of the roof fall } \\
& \mathrm{V} 1=\text { velocity created from a } 6{ }^{\prime} \text { fall } \\
& F=\text { force created from impact } \\
& T=\text { time of impact } \\
& V 2=\text { final velocity (in this case } 0 \text { ) }
\end{aligned}
$$

Force was calculated for a range of impact times. Impact times of 0.15 and 0.30 seconds were chosen to simulate respectively. The forces that were calculated from those impacts as well as the static load of the rocks after the fall were analyzed in a static model. In the model, a trench was excavated and then the cable was laid on a bed backfill and then the trench was filled. A general example of the model geometry can be seen in Figure 4-4. Other parameters analyzed were trench width, ranging from 6" to $12 "$, and trench depth, ranging from 6" to 12" (Terreri, 2008).

Static model results show that for both shale and stiff clay floors there is no apparent correlation between trench width and force observed on the cable. It was also observed that there was a slight correlation between burial depth and force on the cable. Example graphs of these initial results can be seen in Figures 4-5 and 4-6. 


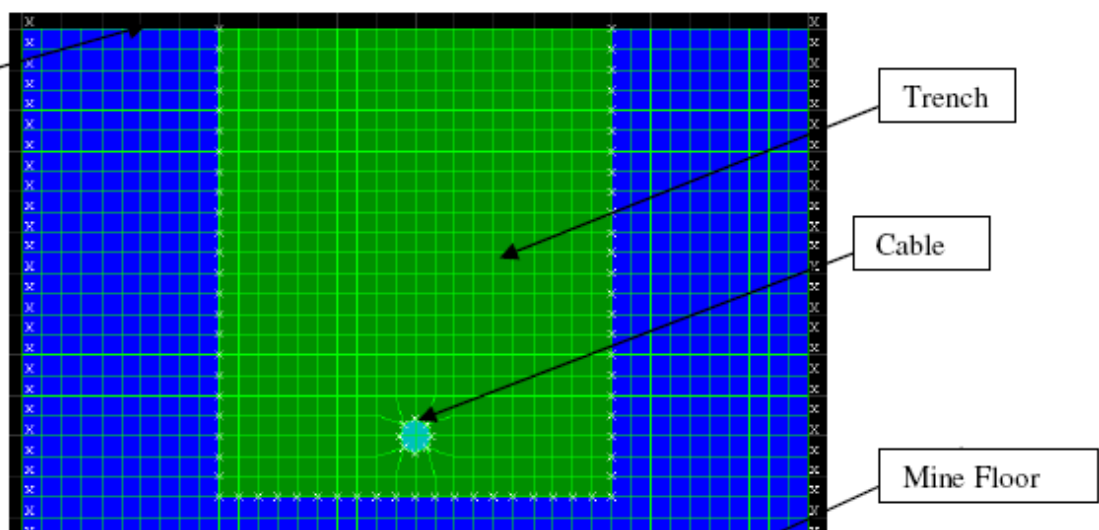

Figure 4-4. Example Model Geometry with 12" Trench Width and 12" Cable Burial (Terreri, 2008).

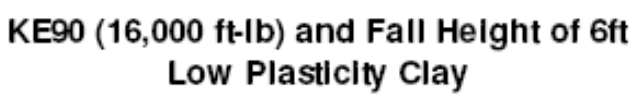

KE90 $(16,000 \mathrm{ft}-\mathrm{Ib})$ and Fall Height of $6 \mathrm{ft}$ Low Plasticlty Clay
Trench Depth: 12"

Cable Bed Thickness: 1.2"

Cable Diameter: 0.5"

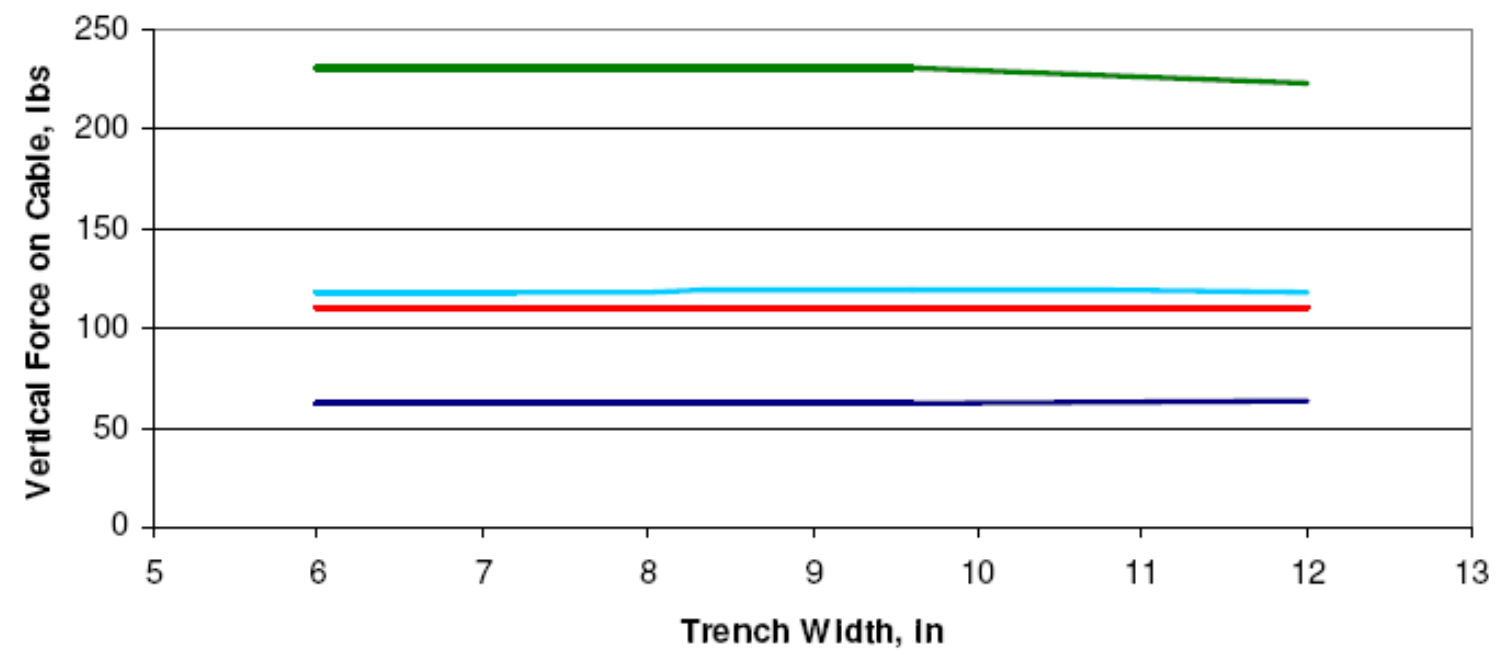

\begin{tabular}{|ll|}
\hline $2,700 \mathrm{lbs}$ Static Load & $5,500 \mathrm{lbs}$ Static Load \\
$11,000 \mathrm{lbs}$ Static Load & Cable Flat Plate Crush Strength \\
\hline
\end{tabular}

Figure 4-5. Preliminary Results from Varying Trench Width (Terreri, 2008). 
KE90 (16,000 ft-lbs) and Fall Height of 6 feet Low Plasticity Clay
Trench Width: 6"

Cable Bed Thickness: 1.2"

Cable Diameter: $0.5^{\prime \prime}$

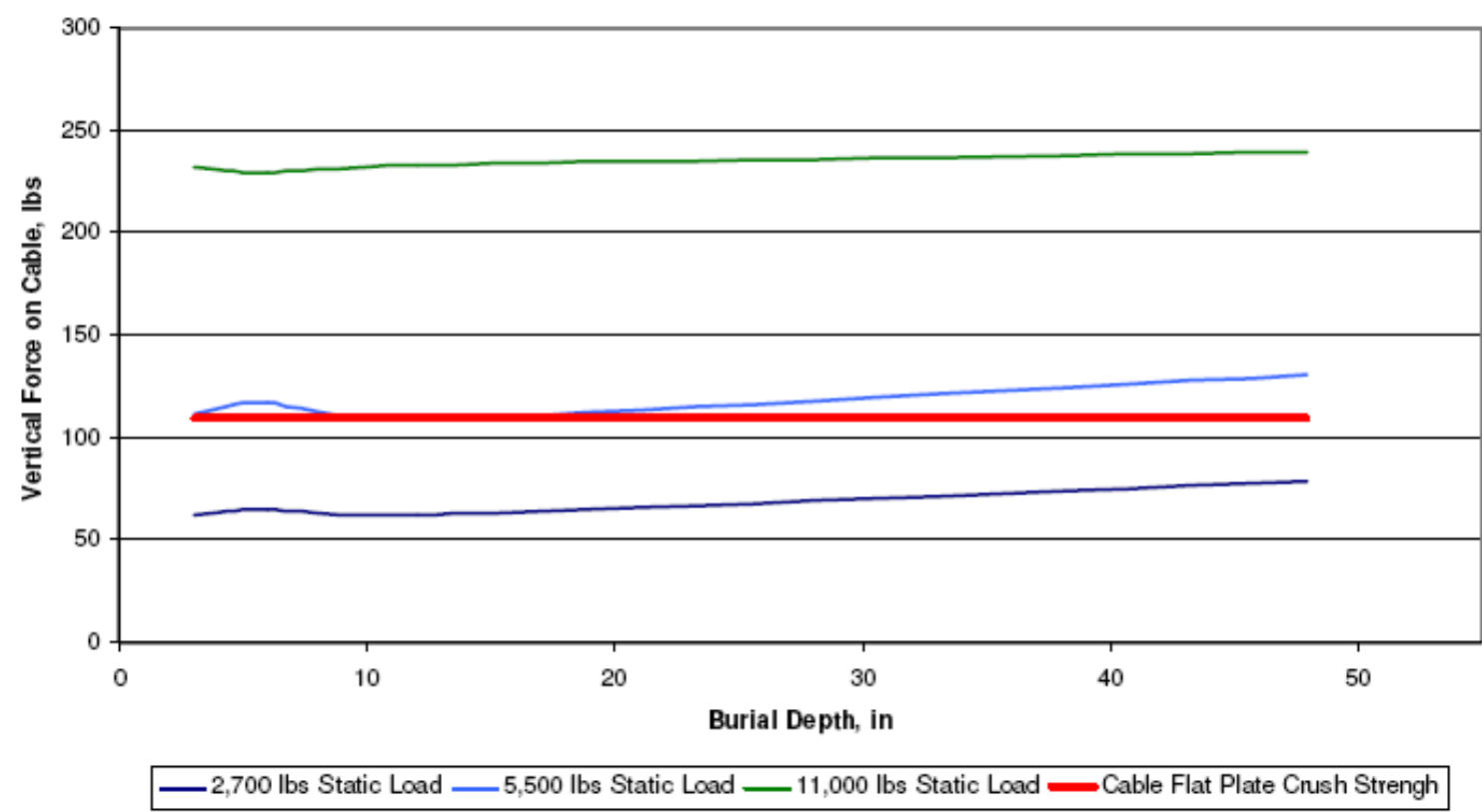

Figure 4-6. Preliminary Results from Varying Burial Depth (Terreri, 2008).

\subsection{ELF / VLF Propagation Modeling}

Much like the other EM models, ELF/VLF models can be very complicated. It is important to keep in mind that these models are made using assumptions that would simplify and generalize the problem. It is important to use the data gathered from the models and compare to the available empirical data.

\subsubsection{Wait's Homogenous Earth Model}

James R. Wait was an internationally recognized expert in EM analysis. Although Dr. Wait has many detailed analyses of TTE propagation, his development of the homogeneous earth model has been used to supply significant insight into the very low frequency (VLF) behavior. In this model, it assumed that the propagation is in an 
infinite earth. There are no reflection or transmission interfaces. The transmitter, an infinitesimal dipole, is assumed to be buried in the earth. Figure 4-7 shows the horizontally oriented transmitter loop of magnetic moment M=NIA (actually an infinitesimal dipole in Wait's analysis) with $\mathrm{N}$ turns, current I and loop area A surrounded by an isotropic homogeneous earth. The magnetic field is desired at point $P$ where the receiver would normally be.

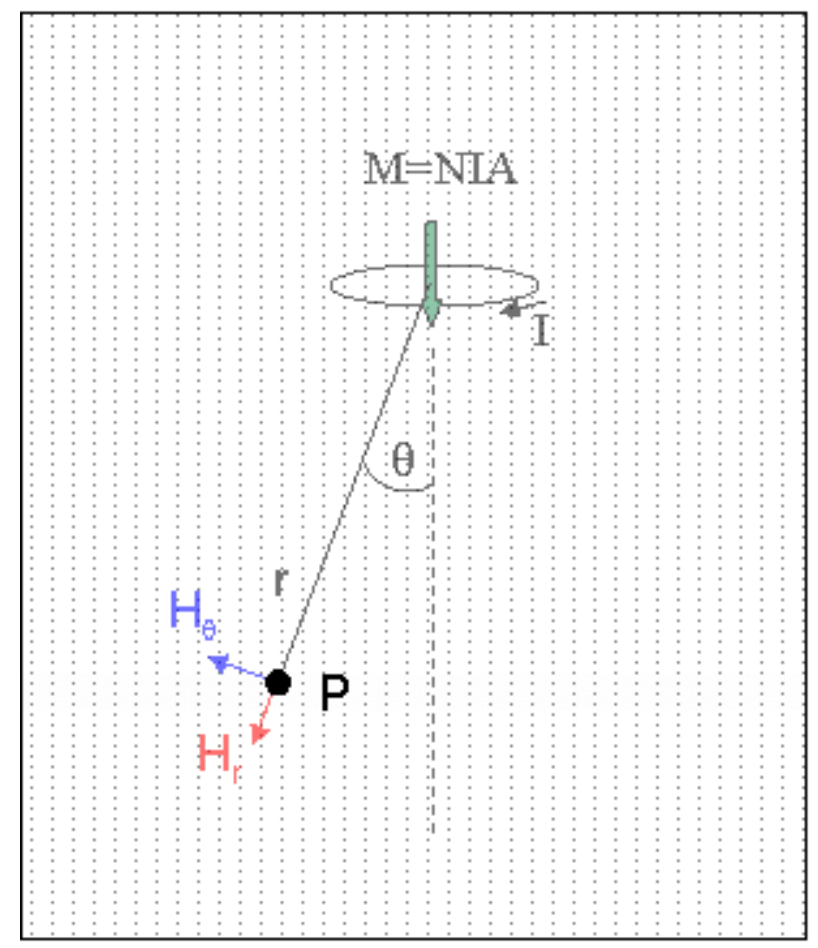

Figure 4-7. A current loop in an isotropic homogeneous earth. The magnetic field components, in spherical coordinates, are desired at a receiver located at point $\mathbf{P}$.

As derived by (Hill and Wait,1982) the 'Maxwell' equations for field strength are presented in equations (17) and (18) for an infinitesimal electric dipole and an infinitesimal magnetic dipole embedded in an infinite homogeneous medium (earth) of electrical conductivity $\sigma(\mathrm{S} / \mathrm{m})$, magnetic permeability $\mu\left(\mathrm{N} / \mathrm{A}^{2}\right)$, and dielectric permittivity 
$\varepsilon\left(A^{2} s^{2} / N^{2}\right)$. The permeability of earth is treated as that of free space, $\mu_{0}=4 \pi 10^{-7} \mathrm{~N} / \mathrm{A}^{2}$. Assuming the time variation is described by $\mathrm{e}^{\mathrm{j} \omega \mathrm{t}}$, where $\omega=2 \pi f$ and $\mathrm{f}$ is the frequency in $\mathrm{Hz}$ and $j=\sqrt{-1}$ where the result in spherical coordinates for an electric dipole of moment $I d l$ with axis vertically oriented along z-axis is the Maxwell equations that follow:

$$
\begin{aligned}
& E_{\theta}=\frac{I d l}{4 \pi \sigma^{3}}\left(1+\gamma r+\gamma^{2} r^{2}\right) e^{-\gamma r} \sin \theta \\
& E_{r}=\frac{I d l}{2 \pi \sigma r^{3}}(1+\gamma r) e^{-\gamma r} \cos \theta \\
& H_{\phi}=\frac{I d l}{4 \pi r^{2}}(1+\gamma r) e^{-\gamma r} \sin \theta
\end{aligned}
$$

and for a magnetic dipole with a loop moment $I d A$ vertically oriented along z-axis

$$
\begin{aligned}
& E_{\phi}=\frac{-j \mu \omega I d A}{4 \pi r^{2}}(1+\gamma r) e^{-\gamma r} \sin \theta \\
& H_{r}=\frac{I d A}{2 \pi r^{3}}(1+\gamma r) e^{-\gamma r} \cos \theta \\
& H_{\theta}=\frac{I d A}{4 \pi r^{3}}\left(1+\gamma r+\gamma^{2} r^{2}\right) e^{-\gamma r} \sin \theta \\
& \gamma=\left(j \sigma \mu \omega-\varepsilon \mu \omega^{2}\right)^{1 / 2} \approx(j \sigma \mu \omega)^{1 / 2}
\end{aligned}
$$

where $\gamma$ is the complex propagation constant.

There are a number of observations that will enhance the understanding of these equations (17) and (18). First, the propagation constant $\gamma$ in (18) can be written as

$$
\gamma=\sqrt{\frac{\sigma \mu \omega}{2}}\left(1+j=\frac{1}{\delta}(1+j)\right.
$$

where $\delta$ is the skin depth. Thus, the product $\gamma * r$ is a distance measured in terms of the number of skin depths. In free space, the analogous quantity is $r^{\star} \beta$ where $\beta=2 \pi / \lambda$, i.e. distances are measured in terms of wave numbers. For propagation in a dielectric like the earth at the low frequencies of interest, the wavelength becomes $2 \pi \delta$, which is 
dramatically reduced compared to its free-space extent. As an example, at $1 \mathrm{kHz}$, the free space wavelength is $300 \mathrm{~km}$. At the same frequency in the earth, with conductivity of $0.01 \mathrm{~S} / \mathrm{m}$, the skin depth is $160 \mathrm{~m}$, and the effective wavelength, $2 \pi \delta$, is $1005 \mathrm{~m}$.

Considerable understanding of radio wave propagation in the earth can be gained by comparing it to propagation in free space. A reasonable range of conductivities for the earth are $0.001<\sigma<0.3$, with $\sigma=0.01 \mathrm{~S} / \mathrm{m}$ as a commonly assumed value. The impedance, over the frequency band of interest, varies from one to three, a factor of at least 100 less than that of free space. Hence, the magnitude of the magnetic field is much greater, a factor of 100 or more, in earth propagation than in free space propagation. This implies that, in earth propagation, most of the energy density is in the magnetic component of the field, $\frac{1}{2} \mu_{o} H^{2}$ rather than in the electric field, $\frac{1}{2} \varepsilon E^{2}$, where $\mathrm{E}=\eta \mathrm{H}$ and $\eta$ is the impedance (Hill and Wait,1982).

Next consider the variation in the strength of the magnetic field as a function of depth below a radiating magnetic loop. As seen in Fig. 4-8, Curve 'a' demonstrates a $1 /$ depth $^{3}$ dependence which can be compared to a near-field dependence on distance (the far field should follow $1 / z$ ) until the exponential begins to dominate as the depth exceeds the corresponding skin depths of $500 \mathrm{~m}, 50 \mathrm{~m}$ and $160 \mathrm{~m}$ for curves b, c, and d respectively. Notice that the field decreases with distance depending on the frequency and conductivity through the value of the skin depth. Thus, the combination of a higher frequency propagating in a lower conductivity earth is equivalent to a lower frequency propagating in a higher conductivity earth as shown in curve $d$ of Figure 4-8. Figure 4-8 also shows that the dependence on the skin depth is not monotonic; curve $\mathrm{c}$ has the 
lowest value of skin depth $(50 \mathrm{~m})$ yet its field strength is intermediate to those of curves a $(\delta=500 \mathrm{~m})$ and $\mathrm{d}(\delta=160 \mathrm{~m})$.

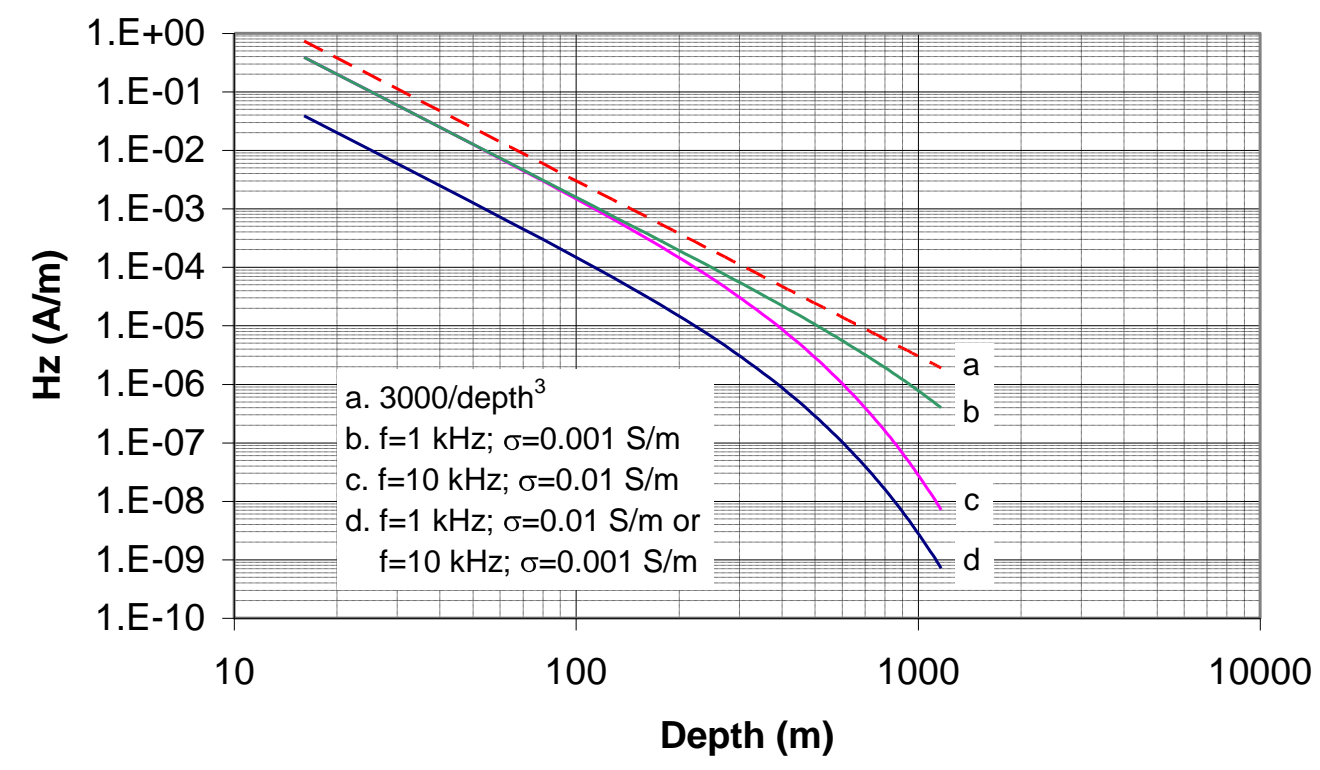

Figure 4-8. Magnitude of the magnetic field directly below a loop of magnetic moment $1000 \mathrm{Am}^{2}$ as a function of depth

In Figures 4-9 and 4-10, the plot shows the components of the magnetic field strength as a function of radial distance from the vertical axis. These plots show that as the radial distance from the vertical axis increases the magnetic field strength decreases, therefore, decreasing reception of communications 


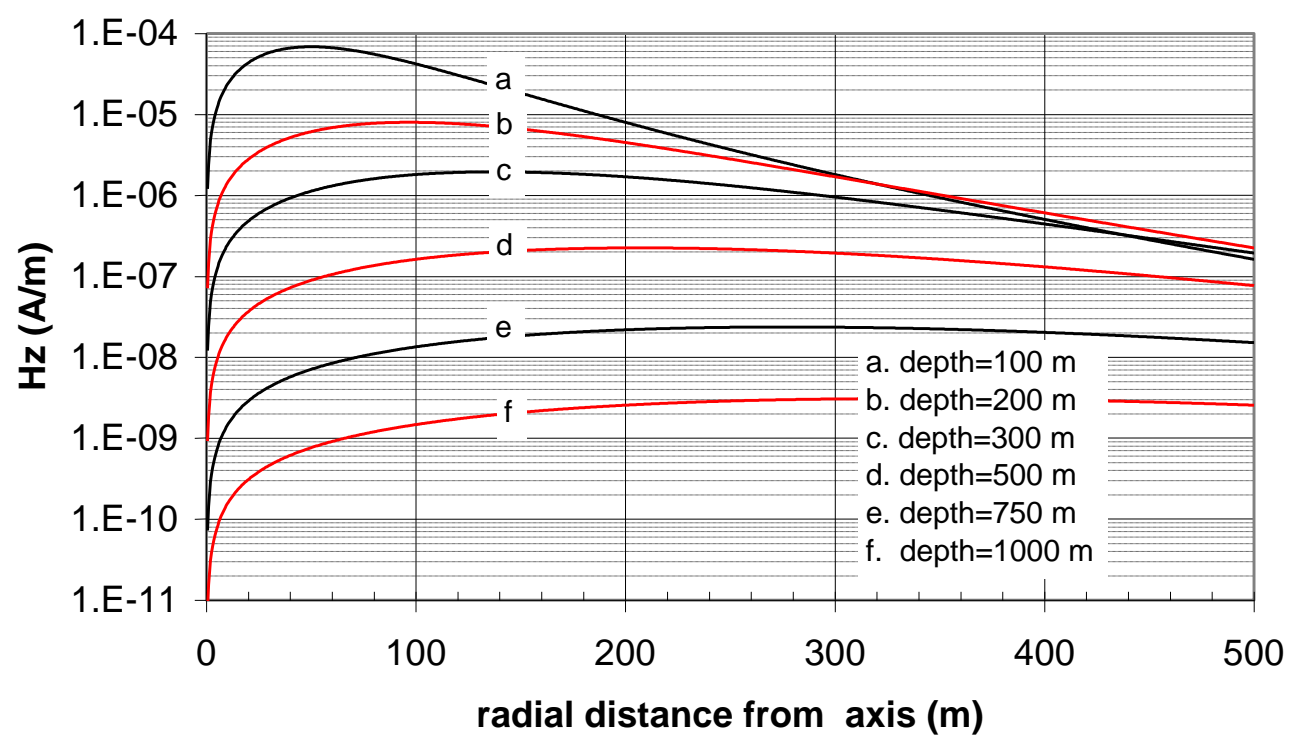

Figure 4-9. Vertical component of magnetic field as function of distance from the vertical axis, assuming a magnetic moment of $1000 \mathrm{Am}^{2}$ oriented horizontally, 1 $\mathrm{kHz}$, and $0.01 \mathrm{~S} / \mathrm{m}$.

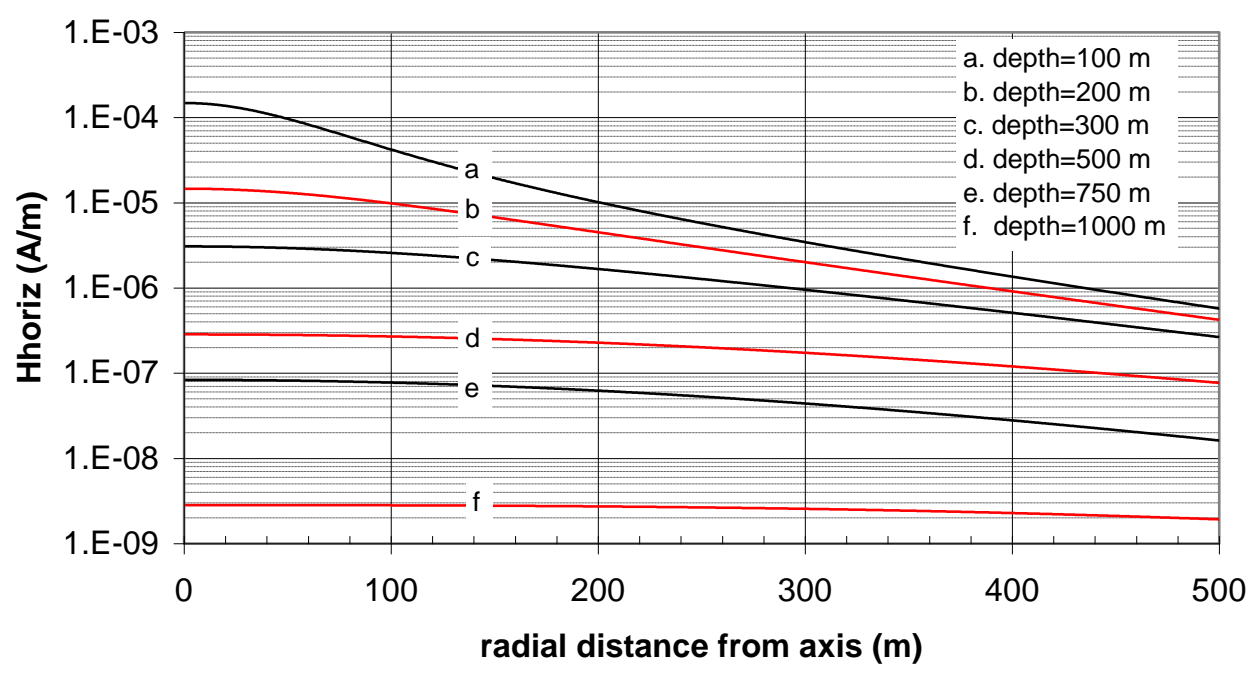

Figure 4-10. Horizontal component of magnetic field as function of distance from the vertical axis, assuming a magnetic moment of $1000 \mathrm{Am}^{2}$ oriented horizontally, $1 \mathrm{kHz}$, and $0.01 \mathrm{~S} / \mathrm{m}$. 
Given the three plots, it can be seen that having the axes aligned is operationally the best case scenario for sending and receiving signals through the earth. However, as the axes deviate and the depth increases the magnetic field strength decreases. Also, this discussion and results assume the relative orientation of the two coils is fixed. This may be likely if the Tx and Rx coils are laid on the ground or one is wrapped around a coal pillar.

\subsubsection{Shope's Three-Layered Earth Model}

One approach to refining the homogeneous earth model is to consider the earth as composed of multiple parallel layers, with each layer having unique electrical parameters. Shope (1982) presented a three-layered earth model with the infinitesimal magnetic dipole (vertically oriented) in the ground (Fig. 4-11)
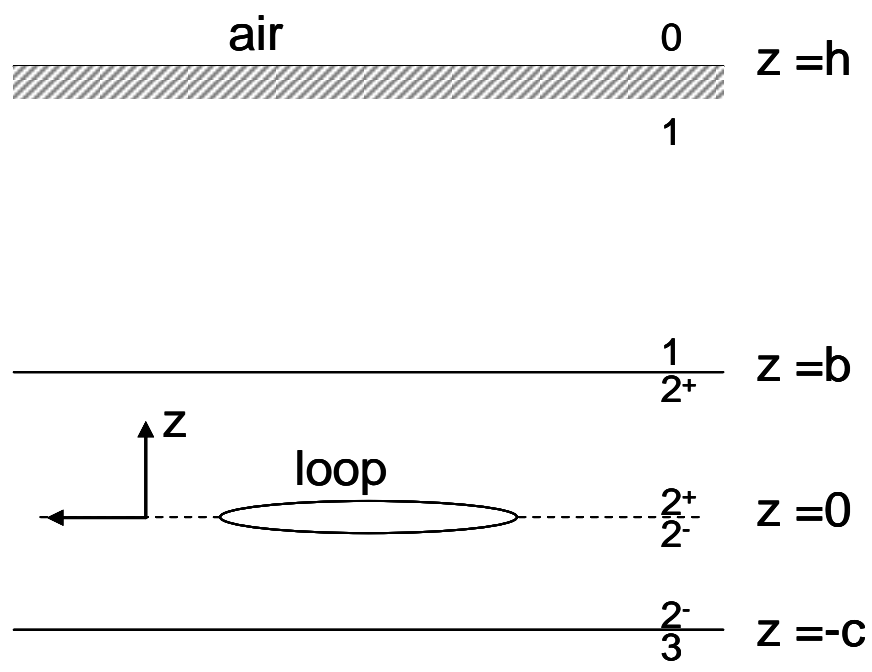

Figure 4-11. Side elevation view of the three-layer earth model. The earth's surface is at the label 'air' at $z=h$. 
Each region is horizontally infinite, isotropic, and homogeneous with unique electrical properties $\sigma$ and $\varepsilon$. The approach is to solve 'Maxwell's' equations using Hertzian potentials (King, 1981). Cylindrical coordinates $(\rho, \phi, z)$ are used. The details of the derivation can be found in Shope (1982). The outputs of interest are the z- and $\rho$ components (vertical and horizontal) of the magnetic field at the earth's surface at $z=h$, labeled as $\mathrm{H}_{z o}$ and $\mathrm{H}_{\rho o}$ respectively. A common convention is to write

$$
H_{z o}=-b Q_{o} \quad \text { and } \quad H_{\rho o}=b P_{o}
$$

where

$$
b=\frac{M}{2 \pi h^{3}} \quad \text { and } \quad M=N I A
$$

and $\mathrm{M}$ is the magnetic moment, the product of the current, I number of turns, $\mathrm{N}$ and area of the loop, A.

Shope (1982) took the further step of normalizing all variables by the depth variable $\mathrm{h}$. Hence, he creates dimensionless variables

$$
\mathrm{D}=\rho / \mathrm{h}, \quad \mathrm{B}=\mathrm{b} / \mathrm{h}, \quad \mathrm{C}=\mathrm{c} / \mathrm{h}, \quad \mathrm{Z}=\mathrm{z} / \mathrm{h}, \quad \text { and } \quad H=\left(\mu \omega \sigma_{2}\right)^{1 / 2} h
$$

where Shope (1982) states that $\mathrm{H}$ is ratio of the source depth $\mathrm{h}$ to the skin depth in region 2 of conductivity $\sigma_{2}$. There appears to be a discrepancy here in that the skin depth is actually defined, as mentioned in the homogeneous earth section, as

$$
\delta=\left(\frac{2}{\mu \omega \sigma}\right)^{1 / 2} .
$$

Hence, there is a factor of $\sqrt{2}$ difference between (22) and (23). The discrepancy appears to be only in the comment and not in the equations he ultimately evaluates, i.e. 
leave the mathematical definition of $\mathrm{H}$ unchanged. Normalized conductivities are also introduced to simplify the analysis.

$$
S_{12}^{2}=\frac{\sigma_{1}}{\sigma_{2}} \quad \text { and } \quad S_{23}^{2}=S_{12}^{2}=\frac{\sigma_{3}}{\sigma_{2}} \text {. }
$$

In addition to the significantly complex equations given by Shope, the model now has more input variables: $S_{12}, S_{23}, B, C, D$, and $Z$. The non-normalized variables are;

- frequency of operation, $f$;

- depth of the coil, h (or possibly run analyses as a function of depth $\mathrm{h}$ );

- conductivities $\sigma_{1}, \sigma_{2}$, and $\sigma_{3}$;

- layer locations, b and c;

- radial distance from coil axis, $\rho$ (or run analyses as a function of $\rho$ ).

In summary, the analysis indicates that the total maximum variation in path loss could be $28 \mathrm{~dB}$ due to the uncertainty in conductivities and layer thicknesses in the three-layer earth model. In comparing a single-layer to a three-layer model, the three layer model yields 10 to $20 \mathrm{~dB}$ greater path loss than a single layer model for what might be termed 'similar inputs.' Therefore, overly simple models and/or data with large uncertainties can contribute to perhaps as much as $50 \mathrm{~dB}$ in path loss. 


\section{Chapter 5}

\section{Empirical and Practical Test}

Empirical analysis is important because the data collected in an analytical model is not always accurate when compared to real world scenarios. Because of this, experiments should be performed and results documented for comparison. Since the Miner Act of 2006, there have been a number of tests and demonstrations done by various vendors, companies, and research agencies. These tests have provided valuable practical data and key insight as to the performance of these systems to be used by the mining industry.

\subsection{WVU test at Lake Lynn Laboratory}

In the Fall of 2006 WVU had the opportunity to conduct tests using the leaky feeder system at the Lake Lynn Laboratory. The goal of the tests was to find out the applicability of a leaky feeder as an emergency communication system. The tests completed are as follows:

- Signal Propagation Test (line of sight)

- Signal Link Penetration and Distribution Test (non-line of sight)

The tests were done from a practical point of view and the conclusions listed reflect that. The conclusions gathered are:

- The leaky feeder system does not intensify the signal, but it spreads the signal throughout the mine.

- The leaky feeder system receives the signal and transmits it throughout the mine at a different frequency through the antenna. 
- If the leaky feeder system stops working due to power failure, the hand held radio will only reach a few hundred meter on its own. So the leaky feeder system, in the absence of a reliable battery back-up, is not a reliable communication system during an emergency.

\subsection{Foundation Coal Leaky Feeder Analysis}

Foundation Coal conducted tests in a number of their mines using different brands UHF and VHF leaky feeder systems. The tests were conducted to find the optimal system for their mines. The following summary of points concludes their findings:

- The Becker VHF system outperformed the Victor VHF system.

- It was demonstrated that underground UHF propagation outperforms VHF propagation by a factor of 2 , independently of the leaky feeder backbone.

- The Becker UHF backbone system outperformed both VHF systems, in terms of lateral coverage off the cable as well as "around the corner" coverage.

- No significant performance difference was noted between the RFS and TRILOGY (Brand Names) UHF radiating cables.

- The UHF amplifiers should ideally be spaced at 300 m intervals.

- The UHF cables can be loosely strapped to the meshing and lacing, but must not be bundled with other cables in trays or hooks.

- UHF Radio data connectivity to fixed and mobile devices was successfully demonstrated through the radiating cable system. 
- The Becker active personnel and vehicle tags were successfully detected and reported to the surface CATS application through the UHF backbone.

- The Becker remote telemetry unit (RTU) successfully measured and reported the results to the surface Adroit SCADA application through the UHF backbone.

- Including a small dipole $3 \mathrm{~dB}$ coupled to the main arterial, in the proximity of a crosscut entrance, substantially improves the crosscut coverage. This should be considered for final system deployments (Allekotte, 2006).

\subsection{Leaky Feeder Explosion Test}

In the summer of $2008 \mathrm{NIOSH}$ conducted several explosion tests of leaky feeder cable at Lake Lynn Laboratory. The test consisted of stringing leaky feeder cable from the roof of the mine in the directions parallel to the entry, as well as perpendicular to the entry. The entry was then sealed and filled with an explosive concentration of methane and ignited. One test specifically, showed that leaky feeder cable is susceptible to high temperatures as well as explosive forces especially when hung perpendicular to the entry as shown in figure 5-4. (Figs. 5-1 to 5-4). 


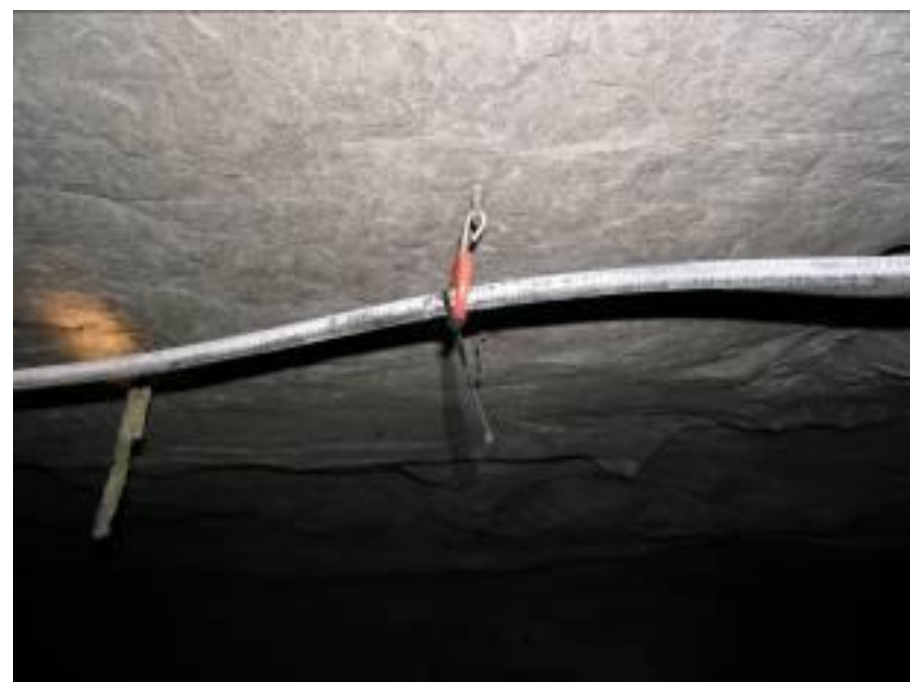

Figure 5-1. Pre-Blast Picture of a leaky feeder cable hung parallel to entry in Lake Lynn Laboratory

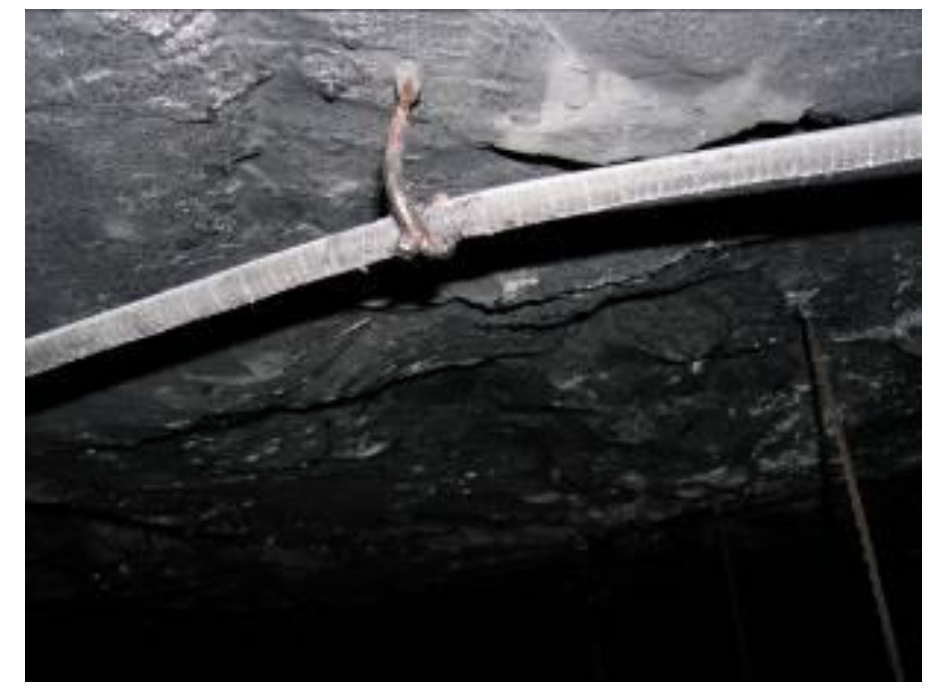

Figure 5-2. Post Blast Picture of a leaky feeder cable hung parallel to entry in Lake Lynn Laboratory (“J” hook melted to cable) 


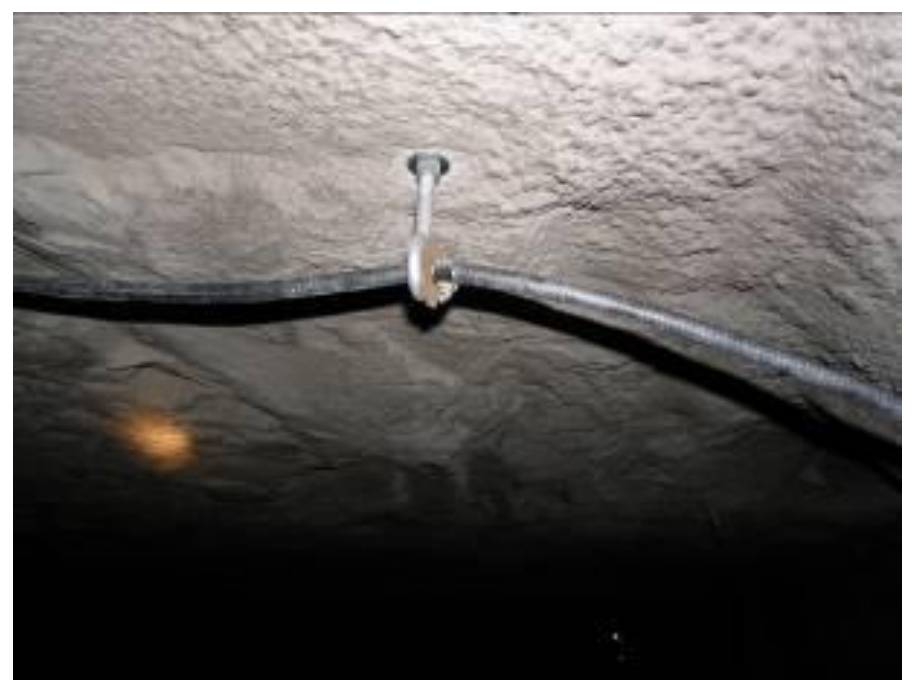

Figure 5-3. Pre-Blast Picture of a leaky feeder cable hung perpendicular to entry in Lake Lynn Laboratory

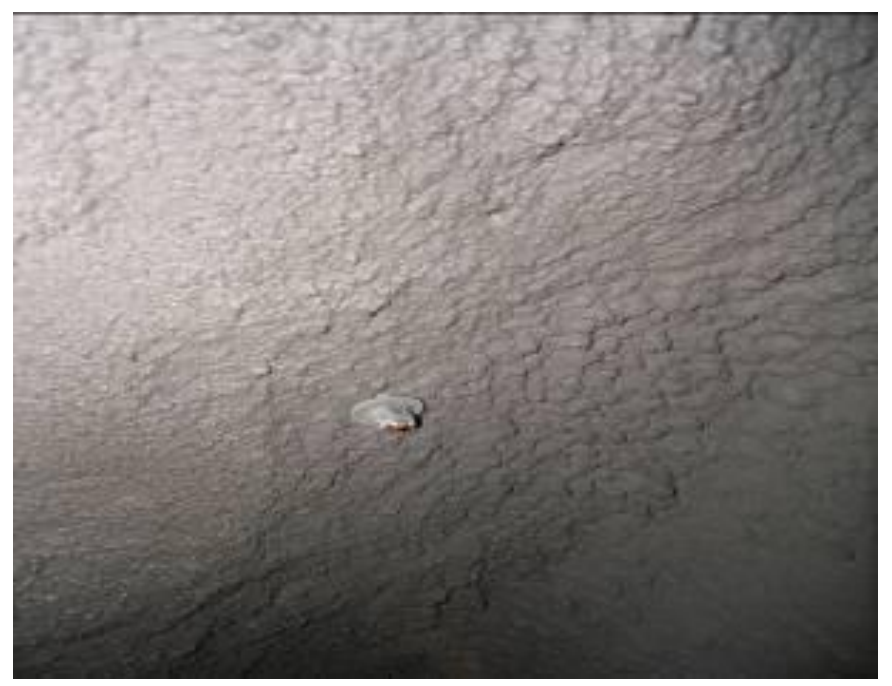

Figure 5-4. Post Blast Picture of a leaky feeder cable hung perpendicular to entry in Lake Lynn Laboratory ("I-bolt” sheared off from explosion, Leaky Feeder destroyed) 


\subsection{MSHA Wireless Communication Study}

The test discussed above centered around leaky feeder systems. In the wake of the Miner Act (2006), MSHA evaluated and performed field testing of six different communication and/or tracking systems. The systems consisted of the following technologies:

- medium frequency radio $(<3 \mathrm{MHz})$

- ultra-wide band radio (UHF/ VHF)

- very low frequency $(<10 \mathrm{kHz})$, through-the-earth

- wireless mesh network (IEEE $802.11 \mathrm{~b}$ or 802.15 .4 standards)

Field test were conducted to determine:

- how well signals propagate (maximum distance between nodes)

- how much overburden systems can penetrate if capable of TTE communication

- mine coverage area (i.e. are there blind spots and why?)

- accuracy of tracking features if interference would be an issue

The tests were conducted at McElroy Mine. A map of the testing area is shown in figure 5-5 (Chirdon, 2006). 


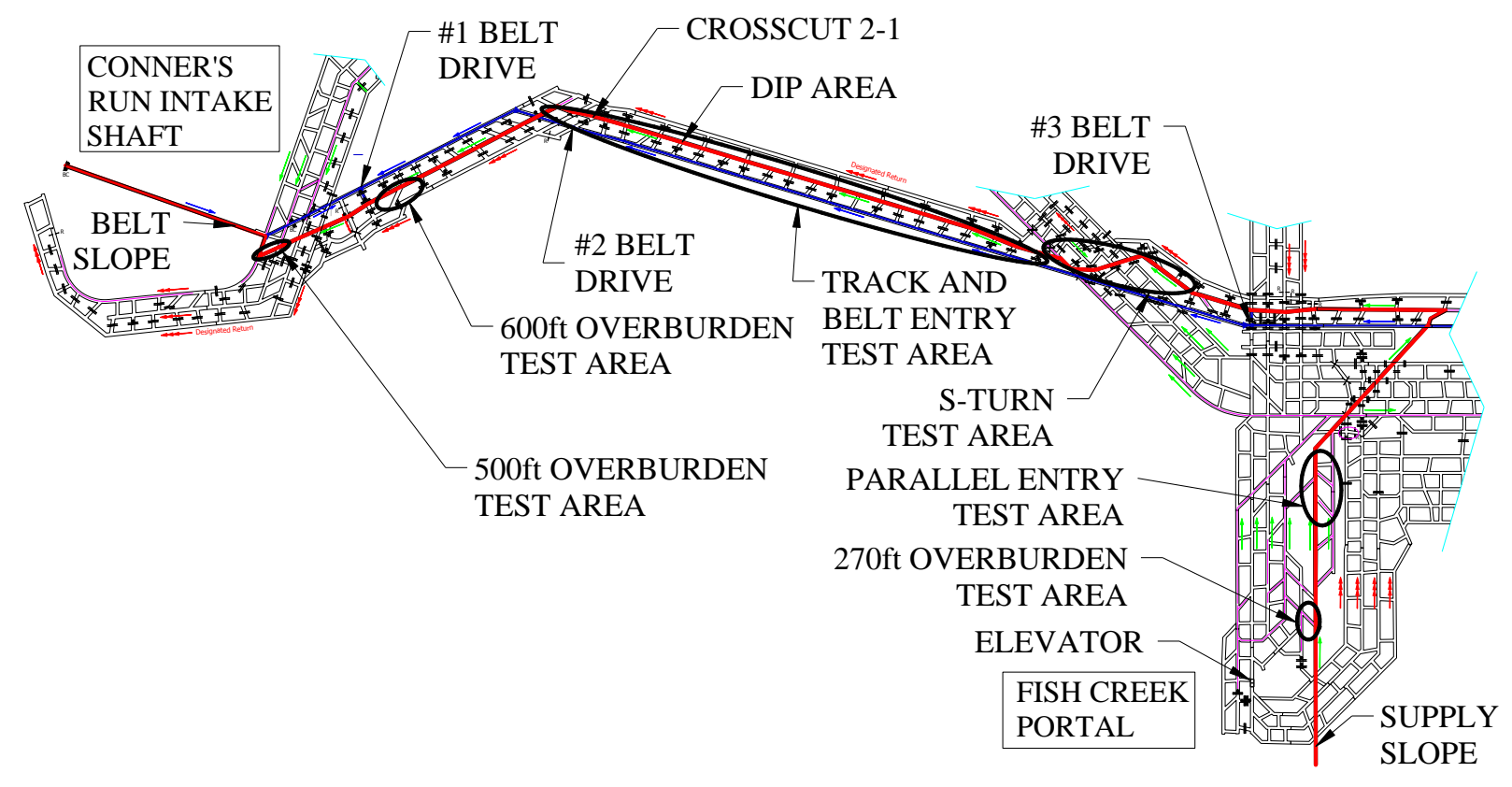

Figure 5-5. Test area McElroy Mine

The results of the test are as follows:

\section{Medium Frequency Radios:}

- Medium frequency radio system signals could propagate more than one mile.

- Systems provide two-way voice and data communications.

- Have interference problem but can be sorted out.

Ultra-Wide Band (UWB) Radio:

- In this test area, range was approximately 1,200 ft with uninterrupted reception and approximately 2,000 ft with some dead spots. The signals produced do not turn corners well; therefore system design must address how to provide coverage in adjacent entries.

- Provide two-way voice communications and tracking with accuracy.

- To ensure that system can function during emergency, access points would have installed in each entry at predetermined distance. 
- Have interference problem but could be sorted out.

Very Low Frequency, Through-The-Earth:

- Signals could penetrate $270 \mathrm{ft}$ overburden with present system but theoretically greater depth can be achieved.

- Also has interference problem.

- Off-axis tests signal could be received.

Wireless Mesh Networks:

- Wireless mesh network type systems that utilize $802.11 \mathrm{~b}$ protocol at $2.4 \mathrm{GHz}$ propagated up to $1,500 \mathrm{ft}$ in this test area.

- The signals produced do not turn corners well; therefore system design must address how to provide coverage in adjacent entries.

- Wireless mesh network type systems that utilize 802.15 .4 protocol at $900 \mathrm{MHz}$ propagated up to $1,800 \mathrm{ft}$ in this test area. The signals produced do not turn corners well; therefore system design must address how to provide coverage in adjacent entries.

- Wireless mesh networks have the potential to provide two-way voice communications and tracking to the nearest node, as well as data transmission.

- In order to outfit the sample test area with communications using wireless mesh network systems, access points would have to be installed in each entry at distances of a maximum of every 1,500 to $1,800 \mathrm{ft}$. Redundancy would also have to be engineered to ensure that the system would continue to function in the event of an explosion or fire. 
- Interference from other communication systems and electrical systems already installed in the mine did not seem to be an issue. The factors that governed signal propagation distance could be attributed to entry geometry in the case of the track entry and both entry geometry and the presence of an abundance of metallic structures in the belt entry

\subsection{Minesite Technologies (TTE DEMO)}

Minesite Technologies is an Australian based company that develops and produces mine communication equipment. Recently they have developed a prototype two-way PED system. The system when functioning correctly can send text messages from underground to the surface and vice versa. The hardware consists of a loop antenna, a transmitter, a receiver, and a laptop PC. The device operates at relative low power in the ELF and ULF EM bands. The device uses proprietary noise cancelation techniques and digital modulation schemes. Since the development the company put on several demonstrations at various mines with varying depths of cover. The device operated successfully at Dana Mining's 4-West Mine (270 ft of cover), Patriot Coal's Federal \#2 Mine ( $800 \mathrm{ft}$ of cover), and Consol's Buchanan Mine (1800 ft of cover). However at 4-west the initial set up for the demo was too close to the surface substation causing too much noise interference. 


\section{Chapter 6}

\section{Conclusions and Recommendations}

From the extent of this research, and the background surrounding it, it is concluded that wireless mine communications has not yet reached its full potential. It can be seen in the analytical section of this thesis that some frequencies used in the mine are not optimal for wave propagation in the entryways. More experimentation with various frequency bands and noise cancellation techniques is needed to optimize these systems. Obviously, some frequency bands are better than others for certain applications. Below is a breakdown of conclusions and recommendations.

\subsection{Recommendation of a Risk Assessment for Battery Back-ups}

All of the systems previously discussed require power to operate. However, in order to operate wirelessly in the case of a disaster, then each system will require battery back-up. The use of batteries also poses interesting problems in the area of maintenance. If each system requires batteries then each system will need to be maintained and checked in order to ensure the batteries are charged and safe. This could be cumbersome due the quantity of batteries that could be needed (depending on what system is being used and the mine layout). Obviously disconnecting the node from the battery would cause a loss of communication; therefore a risk analysis of the use of batteries should be conducted. Currently battery back-up systems have been addressed by MSHA program policy letter P08-V-08. That letter can be seen in appendix A-5. 


\subsection{Leaky Feeder Systems}

These systems have proven themselves capable to supply mine wide communications with the exceptions of a few "dark spots" that are out of range for the leaky feeder (due to corners created by crosscuts). However these types of systems have several drawbacks in the event of an emergency.

- In the event of an emergency the power would be shut off and since the leaky feeder system requires power to operate it would become useless in the absence of a battery back-up. Leaving only the hand held radios to provide communication

- The current hand held radios can only reach approx. $600-1000 \mathrm{ft}$. (line of site) on their own.

- If in the event of a roof fall the leaky feeder cable would most likely become damaged. This would make leaky feeder communication beyond the roof fall impossible. The hand held UHF/VHF radios would most likely not penetrate the roof fall rock.

- As seen in the leaky feeder explosion test conducted by $\mathrm{NIOSH}$ the infrastructure cannot with stand mine fires or mine explosions.

Because leaky feeder systems cannot survive most crisis situations, system hardening as well as burying the cable in a trench, like the one analyzed by Raytheon in Chapter 5 for medium frequency systems, should be researched. If these systems cannot be strengthened then their use as a form of emergency communication should be reconsidered. 


\subsection{Medium Frequency Systems}

Medium frequency systems have a lot of potential in the sence that their ability to use the conductors in the mine can make it possible for the system to survive roof falls, mine fires, and/or mine explosions. Any further development of this technology is highly recommended.

In this thesis models for the coupling of MF transmit antennas to parasitic or deliberate transmission lines, propagating the resulting voltage and current waves down the transmission lines have been discussed. The intent of this effort was to show the feasibility of using standard transmission line modeling techniques to model MF propagation along conductors in coal mines.

The most general approach to the problem would be to build an analytical multiconductor transmission line model that would include several conductors (trolley wires, mine train rails, water lines and so on) and would include branches where the entries split. The transmission line voltage and current sources induced by MF transmit antennas would be inserted as a vector of lumped sources. Receive antenna locations would also be specified. The local fields at the receive antenna locations would be determined from the voltages and currents on the transmission line at the location of interest assuming a quasistatic model.

It is recommended that the next step would be to complete the construction of the integrated end-to-end single conductor (plus return) model with the transmitting and receive antennas included. The resulting code would run in Fortran and would serve as a basis to design the Graphic User Interface (GUI) for the final product. Then the more general multi-conductor model would be written. It is also recommended that the 
models presented here be compared to propagation experiments in an actual mine environment. This would serve to identify any obvious shortcomings in the models and would better quantify the uncertainties in the transmission line approach. Finally it is recommended that the transmission line model be run at VHF/UHF frequencies to see if the transmission line mode is competitive with waveguide models for coal mine entries.

\subsection{Through The Earth Systems}

Through the earth systems can be considered the most sought after piece of technology in terms of mine communications. In order to overcome the strong noise interference, at the frequencies that are required for the system to operate, further development of noise cancelation techniques is recommended.

The homogeneous earth model is very useful for understanding the fundamental behavior of radio wave propagation in the earth. However, it does not allow us to see the effects of varying geology, water table, and pervious mining. The following conclusions for the homogeneous model are presented:

- The controlling variables are frequency, conductivity of the earth, strength of the electric or magnetic moment, orientation of the transmitting moment, orientation of the receiving moment, depth, and radial distance from the axis.

- Lower frequencies propagate with less loss, but the reduced bandwidth will limit the amount of information that can be transferred and/or the time required to transmit the information.

- Lower values of earth conductivity have reduced path loss.

- It can be expected six to eight orders of magnitude decrease in field strength (120 to $160 \mathrm{~dB}$ path loss) in going through $1000 \mathrm{~m}$ of earth. 
- Low values of intrinsic impedance of the earth imply that most of the RF energy is carried in the magnetic field.

- The measured field strength is generally greatest if the transmitter antenna and the receive antenna are horizontally oriented and directly above or below one another, but, operationally, it will be difficult to ensure this alignment. 


\section{Appendicies A}

\section{Appendix A-1 MSHA Approved Wireless Communication Devices}

(December 2, 2008)

\section{Handheld two-way radios}

\begin{tabular}{|c|c|c|c|}
\hline Manufacturer & Model Number & Product Type & Approval \# \\
\hline Kenwood USA Corporation & TK-290, TK-390 & $\begin{array}{l}\text { VHF or UHF } \\
\text { Portable Radio }\end{array}$ & $23-A 060002-0$ \\
\hline $\begin{array}{l}\text { Venture Design Services, } \\
\text { Inc. }\end{array}$ & $\begin{array}{l}\text { Model TMLT } \\
\text { Text Messaging } \\
\text { Location } \\
\text { Transponder }\end{array}$ & $\begin{array}{ll}\text { Tracking } & \text { Tag } \\
\text { and } & \text { Text } \\
\text { Messaging } & \\
\text { Device } & \\
\end{array}$ & 23-A080002-0 \\
\hline Motorola & HT750 & $\begin{array}{l}\text { VHF or UHF } \\
\text { Portable Radio }\end{array}$ & 23-A080007-0 \\
\hline $\begin{array}{l}\text { Alion Science and } \\
\text { Technology }\end{array}$ & $\begin{array}{l}\text { ASM100001 } \\
\text { Accolade }\end{array}$ & $\begin{array}{l}\text { Mesh Radio } \\
\text { Handset }\end{array}$ & 23-A080020-0 \\
\hline
\end{tabular}

\section{Leaky Feeder Communication Systems}

\begin{tabular}{|l|l|c|}
\hline \multicolumn{1}{|c|}{ Manufacturer } & \multicolumn{1}{c|}{ Model Number } & Approval \# \\
\hline Mine Radio Systems & $\begin{array}{l}\text { Flexcom Communications } \\
\text { Systems }\end{array}$ & 9B-219 \\
\hline Varis Mine Tech. & $\begin{array}{l}\text { Model IS Leaky Feeder } \\
\text { Communication System }\end{array}$ & 23-A050001 \\
\hline DAC & Type RFM 2000 Radio System & 9B-201 \\
\hline EL-EQUIP, INC & Model VHF-1 Radio System & 9B-196 \\
\hline $\begin{array}{l}\text { Tunnel Radio of } \\
\text { America }\end{array}$ & $\begin{array}{l}\text { Model UltraComm Distributed } \\
\text { Antenna Communication } \\
\text { System }\end{array}$ & 23-A070005-0 \\
\hline $\begin{array}{l}\text { Becker Electronics } \\
\text { (PTY) LTD }\end{array}$ & Becker Leaky Feeder System & 23-A080003-0 \\
\hline
\end{tabular}

\section{Paging/Text Messaging Systems}

\begin{tabular}{|l|l|l|}
\hline \multicolumn{1}{|c|}{ Manufacturer } & \multicolumn{1}{c|}{ Model Number } & \multicolumn{1}{c|}{ Approval \# } \\
\hline Mine Site Technologies & Model PED1 & 6D-46-0 \\
\hline Mine Site Technologies & $\begin{array}{l}\text { ICCL Integrated } \\
\text { Communications Cap Lamp } \\
\text { with Optional PED }\end{array}$ & 23-ISA080002-0 \\
\hline NL Technologies & $\begin{array}{l}\text { Model GII Cap Lamp } \\
\text { Messenger Circuit }\end{array}$ & 23-ISA070004-0 \\
\hline
\end{tabular}


\begin{tabular}{l|l} 
Stolar Horizon, Inc. & RGU104-001 Remote
\end{tabular}

23-A070002-0

Graphical User Interface

\section{Wireless Mesh Communications and/or Tracking Systems}

\begin{tabular}{|l|l|l|l|}
\hline \multicolumn{1}{|c|}{ Manufacturer } & \multicolumn{1}{|c|}{ Model Number } & \multicolumn{1}{|c|}{ Product Type } & \multicolumn{1}{c|}{ Approval \# } \\
\hline $\begin{array}{l}\text { Venture Design } \\
\text { Services, Inc. }\end{array}$ & $\begin{array}{l}\text { MineTracer Miner } \\
\text { Location } \\
\text { Monitoring System }\end{array}$ & $\begin{array}{l}\text { IEEE 802.15.4 Text } \\
\text { Messaging and } \\
\text { Tracking System }\end{array}$ & 23-A080001-0 \\
\hline NL Technologies & $\begin{array}{l}\text { Digital } \\
\text { Communications } \\
\text { System }\end{array}$ & $\begin{array}{l}\text { IEEE 802.11 mesh } \\
\text { network over fiber } \\
\text { System }\end{array}$ & 23-A080010-0 \\
\hline $\begin{array}{l}\text { Innovative } \\
\text { Wireless } \\
\text { Technologies }\end{array}$ & $\begin{array}{l}\text { Fixed Mesh Node, } \\
\text { with Antenna \& } \\
\text { Battery }\end{array}$ & Fixed Mesh Node & 23-ISA080005-0 \\
\hline $\begin{array}{l}\text { L-3 } \\
\text { Communications }\end{array}$ & Accolade & $\begin{array}{l}\text { Wireless Mesh } \\
\text { Communication } \\
\text { System }\end{array}$ & 23-A080015-0 \\
\hline
\end{tabular}




\section{Appendix A-2 Miner Act (2006)}

\section{Section 2 (F) PLAN CONTENT-SPECIFIC REQUIREMENTS.-}

"(i) IN GENERAL.--In addition to the content requirements contained in subparagraph (E), and subject to the considerations contained in subparagraph (C), the Secretary may make additional plan requirements with respect to any of the content matters.

"(ii) POST ACCIDENT COMMUNICATIONS.--Not later than 3 years after the date of enactment of the Mine Improvement and New Emergency Response Act of 2006, a plan shall, to be approved, provide for post accident communication between underground and surface personnel via a wireless two-way medium, and provide for an electronic tracking system permitting surface personnel to determine the location of any persons trapped underground or set forth within the plan the reasons such provisions can not be adopted. Where such plan sets forth the reasons such provisions can not be adopted, the plan shall also set forth the operator's alternative means of compliance. Such alternative shall approximate, as closely as possible, the degree of functional utility and safety protection provided by the wireless two-way medium and tracking system referred to in this subpart. 


\section{Appendix A-3 List Underground Wireless Communication system requirments in the state of WV (WVOMSHT, 2008).}

\section{TITLE 56 SERIES 4 \\ OFFICE OF MINERS' HEALTH, SAFETY AND TRAINING \\ EMERGENCY RULES GOVERNING PROTECTIVE CLOTHING AND EQUIPMENT}

\section{§56-4-9. Wireless Emergency Communication and Tracking/Locating systems.}

9.1 The Director shall require, in each underground mine, an integrated communication and tracking/locating system maintained consistent with WV CSR Title 36, Series 5 3.2 and a component of which shall be a communication center monitored at all times during which one or more miners are underground. A wireless emergency communication and tracking/locating device approved by the Director shall be worn by each miner underground and shall be provided by the operator.

9.2 As soon as practicable, the Director shall notify all operators of the wireless emergency communication and tracking/locating devices approved by the Director for use by each miner underground pursuant to WV Code Chapter 22A, Article $2-55$.

9.3 The Director shall acquire, no later than July 1, 2006, the necessary technical/engineering support to evaluate the performance of individual communication/tracking devices and review the effectiveness of proposed communication/tracking plans.

9.4 The Director shall, no later than July 10, 2006, issue an open opportunity for emergency communication and tracking/locating providers to submit products for approval.

9.5 The Director shall require providers seeking approval submit documentation certified by a license West Virginia professional engineer that the product has been tested for functionality in West Virginia underground mines, that the product has been or is in the process of being approved as intrinsically safe by MSHA and other criteria as the Director determines, a description of the process used in making that determination and a certification in the following form: "I, the undersigned, hereby certify that this product, to the best of my knowledge and belief, meets or exceeds all requirements set forth in WV CSR Title 56, Series 4-9", that the product has been tested for functionality in West Virginia underground mines, that the product has been or is in the process of being approved as intrinsically safe by MSHA and other criteria as the Director determines.

9.6 No later than July 31, 2007 all underground mine operators shall submit a communication/tracking plan for approval by the Director in accordance with WV Code Chapter 22A, Article 1-36. The design, development, submission, and implementation 
of the communication/tracking plan shall be the responsibility of the operator of each mine.

9.7 Within thirty (30) calendar days after submission of the communication/tracking plan, the Director shall either approve the communication/tracking plan, or shall reject and return the plan to the operator for modification and resubmission, stating in detail the reason for such rejection. If the plan is rejected, the Director shall give the operator a reasonable length of time, not to exceed fifteen (15) calendar days, to modify and resubmit such plan.

9.8 Within fifteen (15) days of approval by the Director, the underground mine operator shall submit as an addendum to its plan, a copy of any contract, or purchase order, or other proof of purchase of any equipment required to complete the communication/tracking system and for installation and ongoing maintenance.

9.9 The operator shall submit certified progress reports no less frequently than every sixty (60) calendar days until full compliance is achieved.

9.10 If the Director, in his sole discretion, determines that an operator has failed to provide a communication/tracking plan or progress report, has provided an inadequate communication/tracking plan or progress report, has failed to comply with its approved communication/tracking plan or compliance schedule, or has failed to provide a copy of any contract, purchase order or other proof of purchase required under this section, in an effort to delay, avoid or circumvent compliance with WV Code Chapter 22A, Article 2 -55 or this rule, the Director shall issue a cessation order to the operator for the affected mine under WV Code Chapter 22A, Article 1-15.

9.11 In developing the communication/tracking plan and any revisions, the operator shall take into consideration the needs for emergency communications and tracking/locating resulting from accidents as described at WV Code Chapter 22A, Article 2-66(a), physical features of the particular mine, emergency plans, existing communication infrastructure, communications required under WV Code Chapter 22A, Article $1-35(\mathrm{k})$ and $2-42$, and WV CSR Title 26, Series $2-2$ and $5-2$, advances in communication/tracking technologies and any other aspect of the particular mine the operator deems relevant to the development of the communication/tracking plan.

9.12 The proposed communication/tracking plan shall describe the structure and operations of the separate or integrated communication/tracking system(s) and its role in emergency response specific to the mine shall be detailed and submitted to the Director and, once approved, to the mine rescue teams providing coverage with an updated mine rescue program pursuant to WV Code Chapter 22A, Article $1-35(q)$. Copies of the most recent version shall be available at the mine for emergency responders. As changes are made to the system, updated versions shall be submitted to the above. 
9.13 The proposed communication/tracking system shall include the ability for:

(1) A communication center monitored at all times during which one or more miners are underground.

(a) This center shall be staffed by miners holding a valid underground miners certificate, and trained and knowledgeable of the installed communications/tracking systems, monitoring and warning devices, travelways, and mine layout.

(b) Individuals not possessing a valid underground miner's certificate but working full time as a communication center operator on or before May 25, 2006 shall be allowed to continue as communications center operators at that mine provided they will have successfully completed no later than December 31, 2006 a certified 80 hour underground miners apprentice training program, as defined in WV CSR Title 48, Series $2-2.7$ (a), renewed annually pursuant to WV CSR Title 48, Series $2-2.8$ (a) and documentation is available for inspection consistent with WV CSR Title 36, Series $24-5$;

(2) Knowing the location of all miners immediately prior to an event by tracking/locating device in the escapeways, normal work assignments, or notification of the communication center;

(3) Knowing the location of miners in the escapeways after an event providing the tracking system is still functional;

(4) Check-in and check-out with the communication center by miners prior to entrance and exit from bleeders and remote or seldom used areas of the mine (all times shall be logged);

(5) Allowing two-way communications coverage in at least two separate air courses and at least one of which shall be intake;

(6) Maintaining communication/tracking after loss of outside power and maintain function both inby and outby of the accident event site with suitable supply of equipment for rapid reconnection;

(7) Maintain a surface supply of communication/tracking devices for use by emergency rescue personnel;

(8) Allow for communication to surface at all required emergency shelters/chambers;

(9) All miners and likely emergency responders shall be trained in the use, limitations and inter-operability of all components of the communication and tracking/locating system. This shall be incorporated into ongoing required training. All training shall be recorded and made available upon request.

9.14 The operator shall provide a schedule of compliance for the communication/tracking plan, which shall include:

(1) A narrative description of how the operator will achieve compliance with above requirements;

(2) A schedule of measures, including an enforceable sequence of actions with milestones, leading to compliance; and

(3) A statement indicating when the implementation of the proposed plan will be complete. 
9.15 The operator shall provide as attachments to its communication/tracking plan:

(1) A statement of the analysis and evaluation required in developing its plan;

(2) A statement indicating the initial training dates for implementation of the communication/tracking system and how the communication/tracking system will be incorporated in other required training;

(3) A statement regarding how the communications/tracking system will be tested and maintained; and

(4)The name of the person or persons representing the operator, including his or her title, mailing address, e-mail address and telephone number, who can be contacted by the Director for all matters relating to the communication/tracking plan and weekly testing of the system.

9.16 After the Director has approved an operator's communication/tracking plan, the operator shall submit revisions to the communications plan at any time that changes in operational conditions result in a substantive modification in the communication/tracking system. In addition, at any time after approval, the operator may submit proposed modifications or revisions to its plan along with reasons therefore to the Director. Within thirty (30) days after receipt by the Director of any proposed revisions or modifications to the communications/tracking plan, the Director shall either approve or reject the revisions, stating in detail the reasons for such rejection.

9.17 The Director may require modifications to a communication/tracking plan at any time following the investigation of a fatal accident or serious injury, as defined by WV CSR Title 36 Series $19-3.2$, if such modifications are warranted by the findings of the investigation. 


\section{Appendix A-4 MSHA Program Policy Letter P06-V-10}

\section{Emergency Response Plan - Content}

1. Post-accident Communication

When hardwired systems are used to meet the MINER Act requirement for redundant communication between surface and underground personnel, wires should be routed through separate entries or boreholes continuous to the surface. MSHA interprets the term "wireless," as used in the MINER Act, to mean that no wired component of the system exists underground where it may be damaged by fire or explosion. Post-accident communication technology would be considered acceptable if, based on its location in the mine and the history of mine explosions and fires in the mine, it is likely to withstand the event intact. A reasonable timetable for installation should be included in the plan.

This provision applies to all mines except anthracite mines with one intake and one return aircourse. In these mines, the redundant hardwired systems may be placed in the same aircourse. 


\section{Appendix A-5 MSHA Program Policy Letter P08-V-10}

SUBJECT: Approval of Communication and Tracking Devices Required by the Mine Improvement and New Emergency Response Act of 2006 (MINER Act)

\section{Scope}

This program policy letter (PPL) is intended for Mine Safety and Health Administration (MSHA) personnel, equipment manufacturers, repair facilities, underground mine operators, underground independent contractors, miner's representatives, and other interested parties.

\section{Purpose}

This PPL is issued to establish approval guidelines for communication and tracking devices under Title 30 Code of Federal Regulations (30 C.F.R.) Part 23, Telephones and Signaling Devices, to address the provisions of the MINER Act.

\section{Policy}

The following guidelines are being administered by the Approval \& Certification Center when processing applications for approval of communication and tracking products for those underground mines or operations required to have permissible equipment:

- Any component or system used to provide voice, text, or signaling data (e.g., tracking) that is intended to remain operational in the event of an emergency is considered a telephone or signaling device and evaluated under 30 C.F.R. Part 23.

- Line powered devices must be equipped with a standby power source to allow continued operation in the event the line power is lost during an emergency. The standby power source must be capable of providing additional operating capacity (24 hours recommended) based on an $80 \%$ idle time, $10 \%$ transmit time and $10 \%$ receive time, denoted as $80 / 10 / 10$ ratio.

- When operating under standby power, all components of a communication or tracking system must be MSHA-accepted as intrinsically safe, or housed in an MSHA certified explosion-proof enclosure. Communication and tracking system components include any interconnecting cables.

- All cables between communication and tracking components must be MSHAapproved as flame-resistant or enclosed in MSHA-approved, flame-resistant hose conduit.

- Intrinsically safe batteries of portable assemblies that are housed in enclosures too large to be subjected to the MSHA intrinsic safety drop test (greater than 5 $\mathrm{kg}$ ) will be evaluated in accordance with the battery enclosure requirements of $\S \S$ 7.44(a), (b), (d), (e), (f), (h), (l) and (m). 
- Standby power sources that include rechargeable batteries must be designed or equipped with means to mitigate the explosion hazard of battery off-gassing. Examples of available mitigation techniques include venting of the enclosure or automatic de-energization when an explosive gas concentration reaches $20 \%$ of the gas' lower explosive limit.

- The standby power source will be subject to MSHA's "Criteria for the Evaluation and Test of Intrinsically Safe Apparatus and Associated Apparatus" (http://www.msha.gov/techsupp/acc/application/acri2001.pdf) to ensure that it does not create a hazardous condition in the de-energized line power portion of the power supply or in the in-coming line power cable (back-feed protection).

- Any potential for radio frequency interference (RFI) with blasting circuits must be detailed by the approval applicant. The approval applicant must specify the maximum output power, normal operating frequency, and the safe distance from blasting circuits.

- Person-wearable tracking tags are considered portable apparatus and therefore are subjected to the MSHA intrinsic safety drop test. Machine-mounted (asset) tracking tags are subjected to an impact test.

- Cap lamps powering communication and/or tracking related components are required to meet the performance requirements specified in $\S 19.9$ (a) when both the cap light and communication and/or tracking component are in operation. To assure sufficient operational capability in various scenarios, the cap lamp battery should be capable of providing sufficient power to effectively operate the communication and/or tracking component for a period of time beyond the 10hour minimum (4 hours additional recommended).

- Where lightning arrestors for conductors between surface and underground locations are required, system approval documentation must specify the lightning arrestor used to comply with $\S \S 57.12069$ and 75.521 , and to ensure that it does not invalidate the Part 23 approval. 


\section{VITA}

David P. McGraw was born and raised in Oak Hill, West Virginia where he finished high school. After completing his work at Oak Hill High School he entered West Virginia University in Morgantown, West Virginia in the Fall of 2001. He received the degree of Bachelors of Science in Electrical Engineering in May, 2007. In August, 2007, he entered graduate school at WVU to study for MS degree in Mining Engineering.

Permanent Address: $\quad 605$ Rogers St.

Oak Hill, West Virginia 25901 


\section{References}

Allekotte, G. "Test of the wireless information System from Becker at Cumberland Mine", Foundation Coal Inc., Penn. Services Corp.Planning and Engineering Group, Memorandum, May 5, 2006

Allekotte, G. "Test of the wireless information System from Becker at Kingston Mine", Foundation Coal Inc., Penn. Services Corp.Planning and Engineering Group, Memorandum, July 18, 2006

Chirdon, D., "Emergency Communication and Tracking Committee Underground Communication and Tracking Systems Tests at CONSOL Energy INC., McElroy Mine," Mine Safety and Health Administration, June 13, 2006.

Chufo,R.L., Lagace,R.L., and Wilson,R.L., "Medium-Frequency Wireless Radio," Underground Mine Communications (In Four Parts), 4. Section-to-Place Communications, IC 8745, Bureau of Mines Information Circular/1977.

Cook, J.C., "RF Electrical Properties of bituminous Coal Samples," Geophysics, Volume 35, Number 6, December, 1970, pp 1079-1085.

Cory, T.S., "Antenna Design \& Coupling Studies at Medium Frequency for Improved Coal Mine Communications," Purchase Order PO382223, United States Department of the Interior, Bureau of Mines, December 15, 1978.

Hill, D.A., and Wait, J.R., "Theoretical Noise and Propagation Models for Through-theEarth Communication," contract no. J0113058 sponsored by Bureau of Mines, May 1982.

Emslie A, Lagace R, Strong P. "Theory of the propagation of UHF radio waves in coal mine tunnels" [J]. IEEE Transactions on Antennas and Propagation, 1975,23:192-205.

Goddard A., "Radio propagation measurements in coal mines at UHF and VHF," in Proc. Through-the-Earth Electromagnetics Workshop, Colorado School of Mines, Golden, Colo., August 17, 1973, available from Nat. Tech. Inform. Service, Alexandria, Va.

King, R.W.P., Owens, M., and Wu, T.T., "Antennas in Matter", MIT Press, Cambridge, MA (1981).

Landee, R.W., Davis, D.C., and Albrecht, A.P., "Electronic Designers' Handbook," McGraw-Hill, 1957 
Large, D.B., Ball, L., and Farstad, A.J., "Radio Transmission to and from Underground Coal Mines-Theory and Measurement," IEEE Transactions on Communications, March, 1973.

MSHA Emergency Communication and Tracking Committee, Report of Findings Underground Communication and Tracking Systems Tests at CONSOL Energy Inc., McElroy Mine, June 13, 2006

Paul, C.R., "Introduction to Electromagnetic Compatibility," John Wiley and Sons, 1992

Paul, C.R., "Analysis of Multi-conductor Transmission Lines," John Wiley and sons, 1994

Sacks, H.K., Chufo, R.L., Proceedings of the Fourth WVU Conference on Coal Mine Electrotechnology, Aldridge-MD, ed. Morgantown, WV, Aug 2-4, 1978, 1978 Aug; :27-1-27-12

Schiffbauer, W.H. and Mowrey, G.L., "Preliminary Assessment of Communications Systems for Underground Mines for Normal and Emergency Operations," can be found at the NIOSH references web site, http://www.cdc.gov/niosh/mining/pubs/.

Shope, S.M., Electromagnetic Surface Fields Due to a Magnetic Dipole Buried in a Three-Layered Earth, 1982, can be found at the NIOSH references web site, http://www.cdc.gov/niosh/mining/pubs/

Smith, G.S., "Loop Antennas," Antenna Engineering Handbook, 2nd Edition, R. C. Johnson and H. Jasik, edit, McGraw-Hill, 1984.

Stolarczyk, L.G., "Development of Demonstration Hardware for Medium Frequency Mine Wireless Communication System," Document No. 3015/9, United States Department of the Interior, Bureau of Mines, September 29, 1980.

Taylor, C.D., Satterwhite, R.S., and Harrison, Jr., C.W., The Response of a Terminated Two-Wire Transmission Line Excited by a Nonuniform Electromagnetic Field, IEEE Transactions on Antennas and Propagation, November, 1965.

Terreri, H., "Preliminary Results for Task III: Buried Conduit/Wire Analysis," Contract No: 254-2008-M-23921 April, 2008

Vance, E.F., Coupling to Shielded Cables, John Wiley and Sons, 1978.

Wait, J.R. "Electromagnetic Scattering from a Wire Grid Parallel to a Planar Stratified Medium," IEEE Transactions on Antennas and Propagation, pp. 672-675, Sept 1972 
Wait, J.R. "Reflection from a Wire Grid Parallel to a Conducting Plane," Canadian Journal of Physics, vol 32, pp. 571-579, 1954.

West Virginia Office of Miners' Health, Safety and Training (WVOMHST), 2008, "Communication and Tracking." 9 Nov. 2008. <http://www.wvminesafety.org/ 